\title{
Physics design of an accelerator for an accelerator-driven subcritical system
}

\author{
Zhihui Li, ${ }^{1,2}$ Peng Cheng, ${ }^{1}$ Huiping Geng, ${ }^{1}$ Zhen Guo, ${ }^{1}$ Yuan He, ${ }^{3}$ Cai Meng, ${ }^{1}$ Huafu Ouyang, ${ }^{1}$ Shilun Pei, ${ }^{1}$ Biao Sun, ${ }^{1}$ \\ Jilei Sun, ${ }^{1}$ Jingyu Tang, ${ }^{1, *}$ Fang Yan, ${ }^{1}$ Yao Yang, ${ }^{3}$ Chuang Zhang, ${ }^{1}$ and Zheng Yang ${ }^{1}$ \\ ${ }^{1}$ Institute of High Energy Physics (IHEP), CAS, Beijing 100049, China, USA \\ ${ }^{2}$ Sichuan University, Chengdu 610064, China \\ ${ }^{3}$ Institute of Modern Physics (IMP), CAS, Lanzhou 730000, China
}

(Received 26 January 2013; published 8 August 2013)

\begin{abstract}
An accelerator-driven subcritical system (ADS) program was launched in China in 2011, which aims to design and build an ADS demonstration facility with the capability of more than $1000 \mathrm{MW}$ thermal power in multiple phases lasting about 20 years. The driver linac is defined to be $1.5 \mathrm{GeV}$ in energy, $10 \mathrm{~mA}$ in current and in cw operation mode. To meet the extremely high reliability and availability, the linac is designed with much installed margin and fault tolerance, including hot-spare injectors and local compensation method for key element failures. The accelerator complex consists of two parallel $10-\mathrm{MeV}$ injectors, a joint mediumenergy beam transport line, a main linac, and a high-energy beam transport line. The superconducting acceleration structures are employed except for the radio frequency quadrupole accelerators (RFQs) which are at room temperature. The general design considerations and the beam dynamics design of the driver linac complex are presented here.
\end{abstract}

DOI: 10.1103/PhysRevSTAB.16.080101

PACS numbers: 29.27.Bd, 29.27.Eg, 29.27.Fh, 41.75.-i

\section{INTRODUCTION}

The China Accelerator Driven subcritical System (C-ADS) project is a strategic plan to solve the nuclear waste problem and the resource problem for nuclear power plants in China. It is supported financially by the central government and administrated by the Chinese Academy of Sciences. With its long-term planning lasting until 2032, the project will be conducted in three major phases: $R \& D$ phase by 2015, experimental phase by 2022 , and demo transmutation facility by 2032 .

The C-ADS accelerator complex is a large $\mathrm{cw}$ proton linac in several sections and uses superconducting acceleration structures except the radio frequency quadrupole accelerators (RFQs), which is under development in collaboration at Institute of High Energy Physics (IHEP) and Institute of Modern Physics (IMP). The main design specifications for the proton beam at the ultimate stage are shown in Table I. Without mentioning the difficulties in the spallation target, the reactor blanket, and the waste separation, to design and build the proton accelerator complex alone is extremely challenging, and there is no existing model in the world.

For the first phase, the project goal is to build a cw proton linac of $50 \mathrm{MeV}$ and $10 \mathrm{~mA}$ by about 2015 . The first phase itself will be executed progressively in several

\footnotetext{
*Corresponding author. tangjy@ihep.ac.cn

Published by the American Physical Society under the terms of the Creative Commons Attribution 3.0 License. Further distribution of this work must maintain attribution to the author(s) and the published article's title, journal citation, and DOI.
}

steps, with the first step to build two 5-MeV test stands of different front-end designs.

The rf frequencies for the main linac have been chosen to follow the Project-X at FNAL, namely, $325 \mathrm{MHz}$ for the spoke cavity sections and $650 \mathrm{MHz}$ for the elliptical cavity sections, in order to share more common design and prototyping results. However, two different designs employing different $r f$ frequencies are pursued for the low-energy part of less than $10 \mathrm{MeV}$, namely, injectors in the technical developing phase, with $325 \mathrm{MHz}$ for Injector Scheme-I and 162.5 MHz for Injector Scheme-II.

For Injector Scheme-I which is proposed by IHEP, the rf frequency of $325 \mathrm{MHz}$ is also close to the frequencies of the previously developed RFQ (352 MHz) for the ADS studies and the actually developing RFQ (324 MHz) for the China Spallation Neutron Sourc (CSNS) project. Much experience on design and fabrication learned from the two RFQs can be useful for the development of the C-ADS RFQ. For Injector Scheme-II proposed by IMP, it was

TABLE I. Specifications of the required proton beam for C-ADS

\begin{tabular}{lcc}
\hline \hline Particle & Proton \\
\hline Energy & 1.5 & $\mathrm{GeV}$ \\
Current & 10 & $\mathrm{~mA}$ \\
Beam power & 15 & $\mathrm{MW}$ \\
rf frequency & $(162.5) / 325 / 650$ & $\mathrm{MHz}$ \\
Duty factor & 100 & $\%$ \\
Beam loss & $<1$ & $\mathrm{~W} / \mathrm{m}$ \\
Beam trips/year [1] & $<25000$ & $1 \mathrm{~s}<t<10 \mathrm{~s}$ \\
& $<2500$ & $10 \mathrm{~s}<t<5 \mathrm{~m}$ \\
& $<25$ & $t>5 \mathrm{~m}$ \\
\hline \hline
\end{tabular}


decided to adopt a lower rf frequency of $162.5 \mathrm{MHz}$ for the RFQ. Lower rf frequency is also well matched to the superconducting half-wave resonator (HWR) cavities that are adopted for Injector Scheme-II. In the following, we will focus on the physical design of Injector Scheme-I and the associated main linac design, and the details about the design of Injector Scheme-II and the associated main linac can be found in Refs. [2-4].

\section{DESIGN PHILOSOPHY AND CONSIDERATIONS}

The C-ADS accelerator complex is designed to have very high beam power and very high reliability, which surpasses those of the existing proton linacs by far. However, several proposed cw proton/deuteron linac projects such as Project$X[5,6]$, EFIT or MYRRHA [7], IFMIF [8] and EURISOL [9], etc. are good models for the physics design and technical design of the accelerator. In addition, some pulsed highpower proton linacs and some cw heavy-ion linacs using superconducting cavities also serve as good reference examples. Although most of the design philosophy for the linac has been addressed by previous literature, we still consider it so important to be stated here.

\section{A. Superconducting cavities as possible}

It becomes a common understanding that the superconducting proton linac is the best choice as an ADS driver accelerator $[1,10]$. With recently approved superconducting rf technology, especially the success of the medium-beta elliptical cavities at SNS $[11,12]$ and the test results of lowbeta spoke cavities $[13,14]$ and HWR cavities $[15,16]$, it is thought that a proton linac with superconducting accelerating structures except the RFQs is possible, which is favored due to the difficulty to deal with huge heat deposit in a cw room-temperature acceleration structure. RFQ as the lowest energy part remains as the only acceleration structure in room temperature. Another advantage of using superconducting cavities is that one can use independently phased resonators to make local compensation [10] when some cavities fail during operation. This is very important to achieve the very strict reliability for ADS accelerators. Certainly, the required rf power that is very important in the cw mode will be largely reduced with superconducting cavities. Even more, the rf power sources based on modular solid-state amplifiers for short superconducting cavities (such as single-spoke cavities) also help to increase the reliability of the linac.

\section{B. Redundancy design}

The most crucial requirement for developing and operating an ADS accelerator is the very high reliability, reflected by the different numbers of beam trips for different durations, as shown in Table I. To achieve the goal, it is important to have installed redundancy for all parts of the accelerator, by means of increasing the reliability of the devices and also the quick online substitution or compensation of device failures [17,18]. Important measures include the maximum use of superconducting acceleration structures, derated parameters for key components, and the local compensation and rematch method for cavity and focusing element failures. For example, in order to meet the requirement of local compensation in case of cavity failures, the nominal operation voltage for the cavities in the main linac section is given with about $1 / 4$ reserve or redundancy. For the very lowenergy part, it is difficult to apply the local compensation method, thus we will design two parallel injectors. When one is in the online operation mode, the other is operated as a hot spare and can be switched to the online mode quickly. Redundant beam position monitors (BPMs) are used to ensure good orbit correction in the case of some failures.

\section{Beam-loss rate control}

Another key point in designing the linac is that beam losses should be kept as low as possible along the linac, with a usual acceptance of $1 \mathrm{~W} / \mathrm{m}$ for all high-power proton accelerators. This is more difficult for the C-ADS, because it has a beam power about 10 times higher than the most powerful existing linac-SNS. This also means a beam-loss rate of $7 \times 10^{-8} / \mathrm{m}$ at the higher-energy part as shown in Fig. 1, and requires very delicate error and beam-loss studies. Thus, it is particularly important to keep low emittance growth along with the acceleration and especially at the matching between different acceleration sections, and strict control of errors and effective orbit correction are needed.

\section{Transition energies for different acceleration structures}

Although more types of acceleration structure or cavity are useful in obtaining efficient acceleration, they also mean more R\&D efforts and higher cost, and this is especially true for superconducting cavities. It should be balanced between the acceleration efficiency and the number of cavity types. Nevertheless, for a large linac such as the

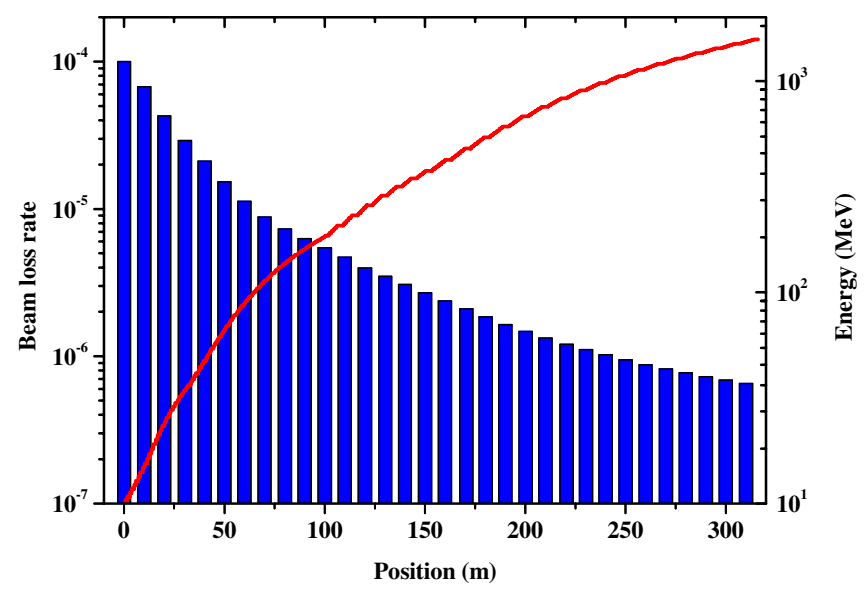

FIG. 1. Maximum allowable lost particles rate per 10 meters in the main linac section. 
C-ADS linac, we need several different acceleration structures and cavity types to reach the final energy of $1.5 \mathrm{GeV}$.

As mentioned before, RFQ is the only room-temperature acceleration structure in the C-ADS linac, and it will be followed by low-beta superconducting cavities. For spoke cavities, higher injection energy is much favored as very low-beta spoke cavities are very difficult to develop and less efficient in acceleration. Because a higher-energy cw RFQ itself is also difficult to develop, the trade-off makes us choose $3.2 \mathrm{MeV}$ as the RFQ output energy, and this is the case of Injector Scheme-I. It is similar for Injection Scheme-II, where a 2.1-MeV RFQ is followed by superconducting (SC) HWR cavities.

For the output energy of the two injectors, on the one hand, one wishes to reduce the total cost by building smaller injectors; on the other hand, higher energy is favored for limiting the emittance growth both in the beam transport section (MEBT2) and in the beginning part of the main linac where the local compensation method of element failures is applied. As a compromise, the injector output energy is set to $10 \mathrm{MeV}$ or slightly higher according to the future optimization. It will be shown later that with a relatively small energy range of $3.2-10 \mathrm{MeV}$ it is possible to design a superconducting section in one cryomodule for the injector.

According to the studies at Project-X [5], single-cell spoke cavities are very efficient in the low and medium energy until about $150 \mathrm{MeV}$. In this design, the output energy of the spoke section in the main linac is about $160 \mathrm{MeV}$ with two types of spoke cavities. With five-cell elliptical cavities, one can reach the final design energy of $1.5 \mathrm{GeV}$ from about $160 \mathrm{MeV}$ with two types of elliptical resonators with the geometrical beta values of 0.63 and 0.82 .

\section{E. Focusing structures}

With the very large energy range and the different acceleration structures, it is less efficient to design the same focusing structure for the whole linac. Different focusing structures in both the transverse and longitudinal planes are used for the best fit to the different acceleration structures or energy ranges. However, we should maintain the smooth variation or better adiabatic change in phase advance per meter, as any abrupt change will result in important emittance growth due to the space-charge effect.

Although the maximum zero-current phase advance per cell is limited to about $90^{\circ}$ to avoid parametric resonances, it should not be lower than about $20^{\circ}$, as higher phase advance is favored to obtain higher acceleration gradient and be less sensitive to the beam current variation. When keeping the phase advance per meter varying adiabatically, jumps in the phase advances per cell in both the transverse and longitudinal planes are needed as the period length is changed abruptly at the transition between two different sections.

For the low-energy part until around $160 \mathrm{MeV}$, superconducting solenoids are considered to be very effective in the transverse focusing. Quadrupoles either in warm sections or in cryomodules will increase the period length that reduces the acceleration efficiency [6]. On the other hand, the beam emittance is almost symmetric in the two transverse planes, so a round beam can be produced to avoid strong emittance coupling between the two planes for the solenoids-based focusing structure. As the defocusing effect of the rf field is evident at low energy, the focusing structure in this energy range appears as different combinations of R (rf field) and S (solenoid), such as SR and RSR.

For the high-energy part, the transverse focusing structure is changed to be quadrupole triplets, as they are more efficient in focusing at higher energy. Because the focusing periods become quite long, the quadrupoles can be placed in the warm sections between the cryomodules. Compared with doublet cells adopted by other designs [5,19,20], triplet cells are favored for their readjustability in case of one quadrupole failure in a cell, and this is very important to minimize the mismatch when keeping very high reliability.

\section{F. Space-charge effects}

Space-charge effects are the dominant factor in inducing the emittance growth in high-intensity linacs. Although the peak current at the C-ADS is significantly lower than most pulsed linacs, its relatively long focusing periods in the low-beta sections have an important impact to tune depression due to smaller phase advance per meter. With a beam current of $10 \mathrm{~mA}$, the tune depression along the linac also reaches about 0.75 which can be considered as emittance dominant, as most people accept the tune depression of 0.71 as the separation between emittance dominant beam and space-charge dominant beam [21]. Figure 2 shows the tune depression along the spoke sections in the main linac.

The importance of the tune depression is also reflected in the Hofmann's chart [22-24]. In order to avoid important resonances induced by the space charge, the linac is designed to follow the resonance-free or equipartition condition approximately. However, for a strong tune depression, higher-order resonances that lead to the emittance change between the transverse and longitudinal phase planes may still happen.

If Injector Scheme-II [2,3] is finally adopted, then we will have doubled bunch intensity in the main linac due to the rf frequency doubling from the injector to the main linac. In this case, the tune depression becomes even smaller, say 0.64 , and the beam becomes space-charge dominant. One must pay more attention to the space-charge effect.

\section{G. Emittance and acceptance}

The beam emittances in the three phase planes are very important design parameters in the C-ADS linac. As the beam-loss rate should be controlled at an extremely low level, all the measures should be considered to control the emittance growth along the linac, especially the halo emittance that is directly linked to the beam loss. 


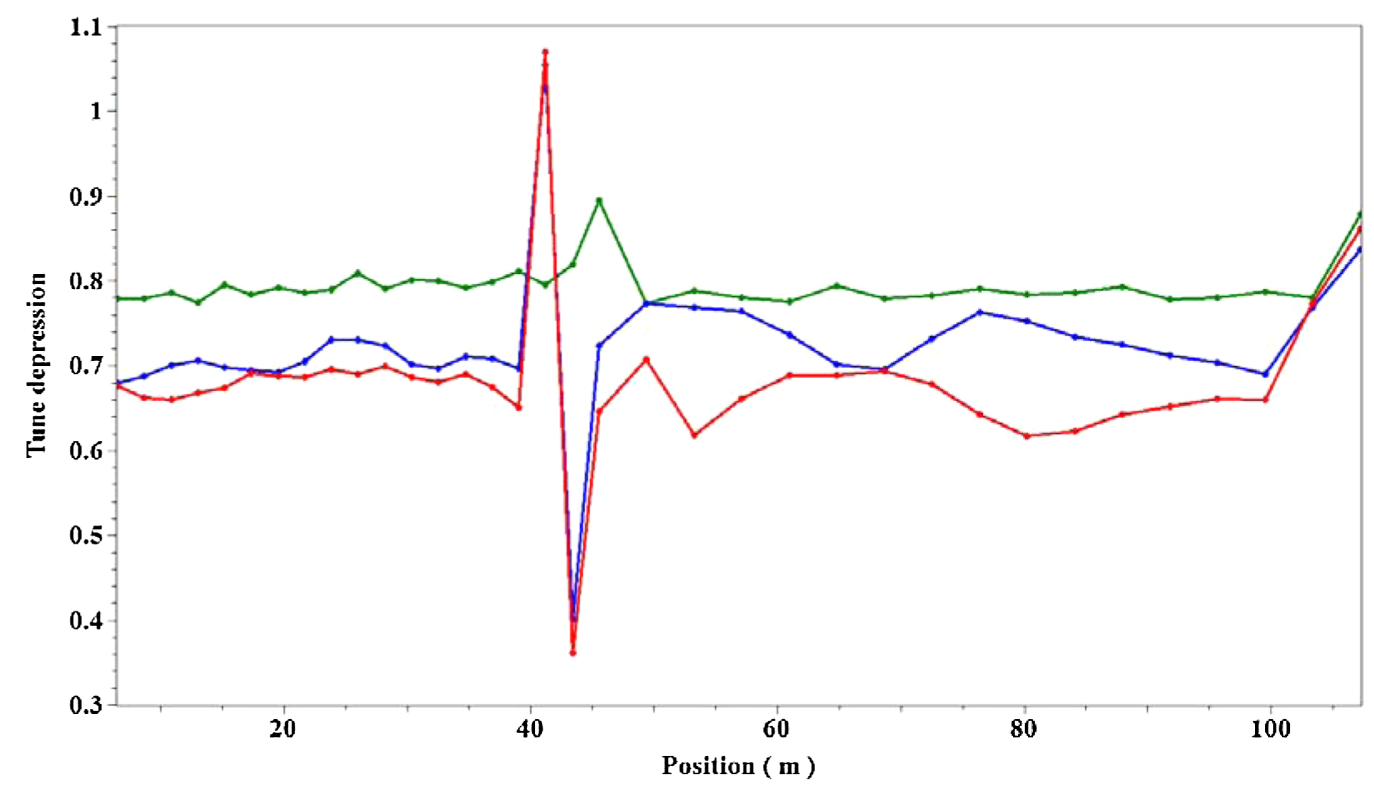

FIG. 2. Tune depression along the spoke sections in the main linac ( $X$ in red, $Y$ in blue, and $Z$ in green).

The initial transverse emittance can be defined by the emittance diaphragm in the low energy beam transport line (LEBT), which is about $0.2 \pi \mathrm{mm} \mathrm{mrad}$ in normalized $\mathrm{rms}$. The longitudinal emittance can be defined by the beam bunching process in the RFQ, which is taken as about $0.18 \pi \mathrm{mm} \mathrm{mrad}$ in normalized $\mathrm{rms}$ as the multiparticle simulation shows. Some halo particles in both the transverse and the longitudinal phase spaces can be collimated in the MEBT2 section.

As mentioned above, the beam is close to be spacecharge dominant, thus emittance parameters are important in the focusing design. In addition, due to the nonlinearity of the rf focusing in the longitudinal phase plane or the rf defocusing in the transverse phase plane it is very important to keep a large ratio between the acceptance and the emittance. For a $6 \mathrm{D}$ water-bag distribution, we keep the acceptance to the full emittance ratio larger than 10 for the whole linac except in the injector part where the ratio is relatively smaller. As the longitudinal acceptance is strongly dependent on the synchronous phase $\left(\phi_{s}\right)$, to keep the same acceptance-emittance ratio, a larger longitudinal emittance means a large $-\phi_{s}$, consequently a lower acceleration rate and a stronger longitudinal focusing. In addition, the phase advance limitation of less than about $90^{\circ}$ in the case of larger $-\phi_{s}$ also reduces the applied rf voltage, and this reduces the acceleration rate further. On the other hand, a larger longitudinal emittance helps increase the tune depression modestly. Therefore, it looks as if it is appropriate to adopt relatively small emittance for both the longitudinal and transverse phase planes.

For the transverse acceptance, it is limited by the nonlinearity of the $\mathrm{rf}$ defocusing at low energy and by the physical aperture of the beam pipes at higher energy. Relatively smaller acceptance will be adopted in the warm sections to avoid beam losses in the superconducting cavities.

Although the geometrical rms emittance will shrink along with the acceleration, it looks as if the total geometrical emittance will still increase during the acceleration due to all the errors and mismatches. Therefore, large physical apertures are still needed at higher energy.

\section{H. Acceleration gradients}

A higher acceleration gradient is usually pursued to reduce the total length or the total investment cost for all kinds of proton linacs. It is due to the same reason to use different acceleration structures at different energy ranges. However, there are some other reasons that we have to reduce the gradient or the applicable rf voltage: (a) at lower energy, due to the limitation on the longitudinal phase advance per cell that should not exceed $90^{\circ}$ significantly. As the bunch length is large here, smaller synchronous phase (negative) is needed to provide a large bucket width, but this will increase the longitudinal focusing simultaneously. Thus the applicable rf voltage is limited. (b) The transit time factor is sensitive to the gap width at lower energy, thus the gap widths for the spoke cavities are optimized to obtain the maximum voltage for a nominal surface electric field. (c) At lower energy, the transverse focusing in the presence of strong space-charge force and rf defocusing requires every cavity or every two cavities intermittent with focusing solenoids. This also reduces the average acceleration gradient. (d) At higher energy, superconducting cavities are much more efficient in acceleration gradient, and the constraints rely mainly on the performance of the superconducting cavities themselves. The focusing becomes much less important, but the grouping of cavities with intermittent focusing elements still has to be considered to maintain the 
smooth change in phase advance per meter and the phase advance per cell always larger than $20^{\circ}$.

\section{Key parameters for the accelerator physics design}

As mentioned before, installed redundancy is required for ADS linacs to obtain very high reliability. According to the state-of-art performance and the prototype experience $[25,26]$, we define some key hardware and physics parameters for the physics design of the C-ADS linac. (1) For all SC spoke cavities: $E_{\text {peak }}<32.5 \mathrm{MV} / \mathrm{m}, B_{\text {peak }}<65 \mathrm{mT}$. (2) For all SC elliptical cavities: $E_{\text {peak }}<39 \mathrm{MV} / \mathrm{m}, B_{\text {peak }}<$ $65 \mathrm{mT}$. (3) Operation temperature for all SC cavities: $1.8 \mathrm{~K}$. (4) Apertures for SC cavities: $35 \mathrm{~mm}$ for $E<10 \mathrm{MeV}$; $40 \mathrm{~mm}$ for Spoke021; $50 \mathrm{~mm}$ for Spoke040; $100 \mathrm{~mm}$ for Ellip063 and Ellip082. (5) Maximum length for cryomodules: $<12 \mathrm{~m}$. (6) rf frequency for the injectors: $325 \mathrm{MHz}$ or 162.5 MHz. (7) rf frequency for the main linac: $325 \mathrm{MHz}$ (spoke sections) and $650 \mathrm{MHz}$ (elliptical sections). (8) Maximum magnetic field for solenoids: 5 T. (9) Beamloss rate: $<1 \mathrm{~W} / \mathrm{m}$. (10) Phase advance per cell (zero current, both transverse and longitudinal): $<90^{\circ}$. (11) Acceptance to emittance ratio (for $6 \mathrm{D}$ water-bag distribution): $>9$.

\section{PHYSICS DESIGN OF DIFFERENT SECTIONS}

The C-ADS accelerator complex consists of two injectors, a special medium-energy beam transport line (MEBT2), a main linac and a high-energy beam transport line (HEBT), as shown in Fig. 3. Two identical injectors will be operated in the mode of one as the hot spare of the other to provide quick switch in case of failure of the one in delivering beam to the main linac. MEBT2 is to transport and match the beam from either of the two injectors to the main linac. As mentioned in the last section, this is crucial to achieve very high reliability of the whole accelerator. However, in the early developing phase two different approaches of injector will be developed in parallel by two different teams. At a later phase, a decision based on the $\mathrm{R} \& \mathrm{D}$ development will be made about which injector scheme will be used in the future phase. The main linac section that consists of different acceleration sections will be developed in phases.
For such a high-intensity linac, the beam dynamic performance with space charge included is very important. Many iterations between the lattice design and the multiparticle simulations have to be performed. The physics design of the C-ADS accelerator complex has been carried out mainly by using the codes PARMTEQM [27], TRACEWIN [28], and TRACK [29]. The 3D field maps are used for all the cavities except the RFQs, and for the solenoids and quadrupoles the element's transfer matrices are used. The 3D field maps of solenoids are used to check the difference with the matrix method, and it is found that the difference is marginal. In the next subsections, the lattice designs for different sections are introduced first and optimized by iterations with multiparticle simulations. The end-to-end simulation results are presented in Sec. III G, and they also affect the global optimization of the linac design.

High-energy beam transport (HEBT) is very important in ADS applications. As the HEBT design for C-ADS is still in the very preliminary stage [4], mainly due to the fact that the interface among the accelerator, target, and reactor has not been clearly defined, it is not presented here.

\section{A. Ion sources and LEBTs}

For a modest beam current about $10 \mathrm{~mA}$ in $\mathrm{cw}$ mode, electron cyclotron resonance (ECR) ion source is a good choice. A similar ion source using permanent confinement magnets was developed at IMP [30]. Although the proton linac for ADS is working in cw mode, the front-end needs to have the ability to provide very short pulse beam as short as $20 \mu$ s for commissioning. After having studied the very short pulse length operation mode for commissioning the superconducting cavities, we found that a chopper with a fast rise/fall time of about $20 \mathrm{~ns}$ in the LEBT is indispensable. The chopper is also important in the machine protection and producing a gap in $\mathrm{cw}$ beam for the $k$-value measurement in the subcritical reactor.

For a very low-energy and high-intensity beam, nonlinear space-charge force is the main cause of beam divergence and emittance growth. Although the residual gas tends to be ionized and the beam space-charge effect is neutralized spontaneously, it is difficult to compensate the space-charge effect completely. Therefore, a shorter LEBT

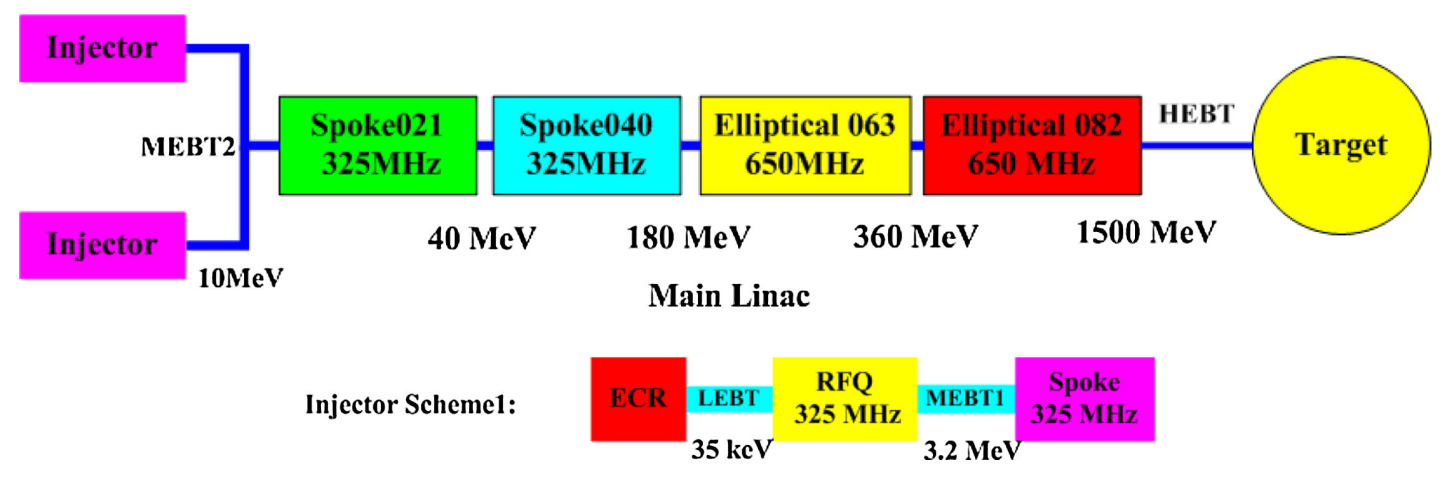

FIG. 3. Layout of the C-ADS driver accelerators. 


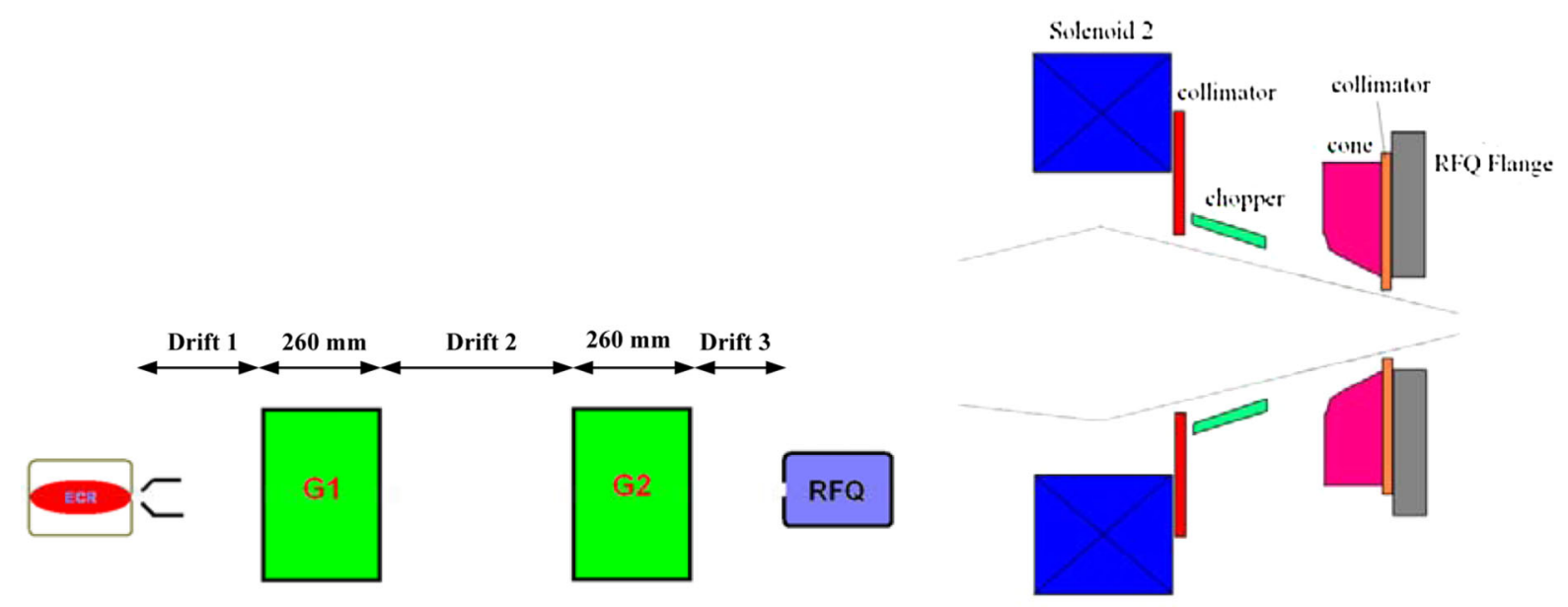

FIG. 4. Schematic for the LEBT (left) and the chopper (right). G1 and G2 are for Glaser magnetic lenses.

is preferred when all the functions such as transverse matching, beam chopping, and emittance shaping, etc., are in place. The chopper is placed at the end of the LEBT to maintain the neutralization effect by following the design at CSNS [31]. The general layout of the ion source and LEBT is shown in Fig. 4.

The TRACE-3D [32], BEAMPATH [33], GPT [34], and TRACK codes were used in designing the LEBT and performing simulations for matching the LEBT to the RFQ. Figures 5 and 6 show the simulated beam trajectories along the LEBT line and the distribution in the phase space at the RFQ entrance. The simulations show that the LEBT design can meet the intensity requirement with the given emittance at the RFQ entrance for both injector schemes.

\section{B. RFQ dynamics design}

The cw RFQ is one of the most critical parts in ADS applications. The design of the RFQ for Injector Scheme-I is based on the following philosophy obtained from the experience of the design and build of the similar machines

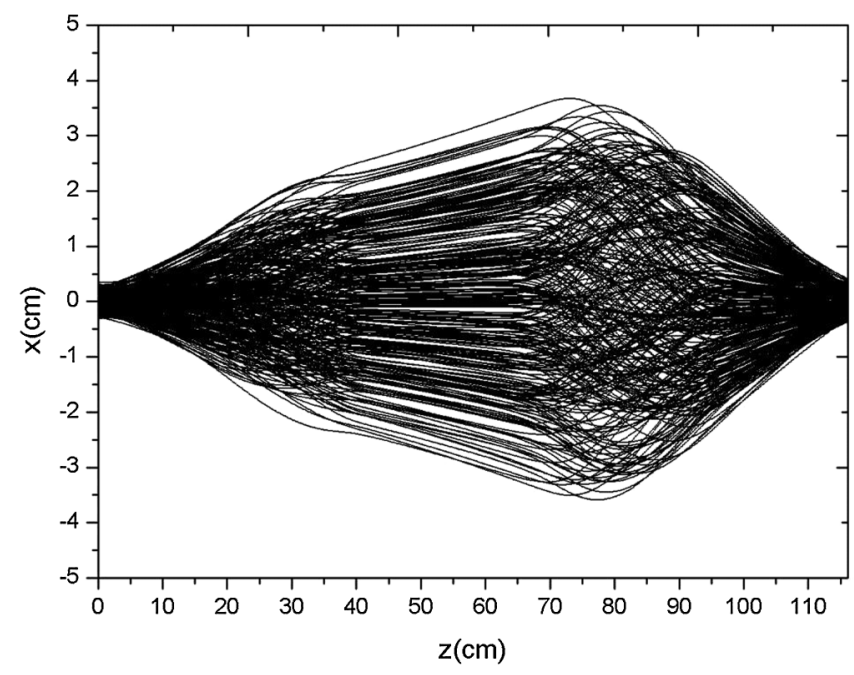

FIG. 5. Trajectories along the LEBT simulated by BEAMPATH.
[35-37]: (1) The key parameters for the RFQ follow the definitions for the C-ADS linac. As the beam current for the linac is $10 \mathrm{~mA}$, the design beam current of the RFQ is chosen to be $15 \mathrm{~mA}$ after taking into account the adjustability and its upgrading potential. The relatively low beam current is also helpful for reducing the required transverse focusing strength. The injection energy is also chosen to be a comparatively lower value of $35 \mathrm{keV}$, to enhance the bunching process in the RFQ and also ease the construction of the ion source and the LEBT while the space-charge effects at $15 \mathrm{~mA}$ in the LEBT are manageable. The output energy is optimized to about $3.2 \mathrm{MeV}$ as a compromise between the technical difficulties of the RFQ fabrication with higher energy and the favorable higher-energy requirement by the low-beta superconducting spoke cavities in the injector. (2) The total length of the RFQ is limited within $4.8 \mathrm{~m}$. The RFQ will consist of two resonantly coupled physical segments and each segment includes two technical modules connected together with flanges. The length of the technical module is limited to $1.2 \mathrm{~m}$ by

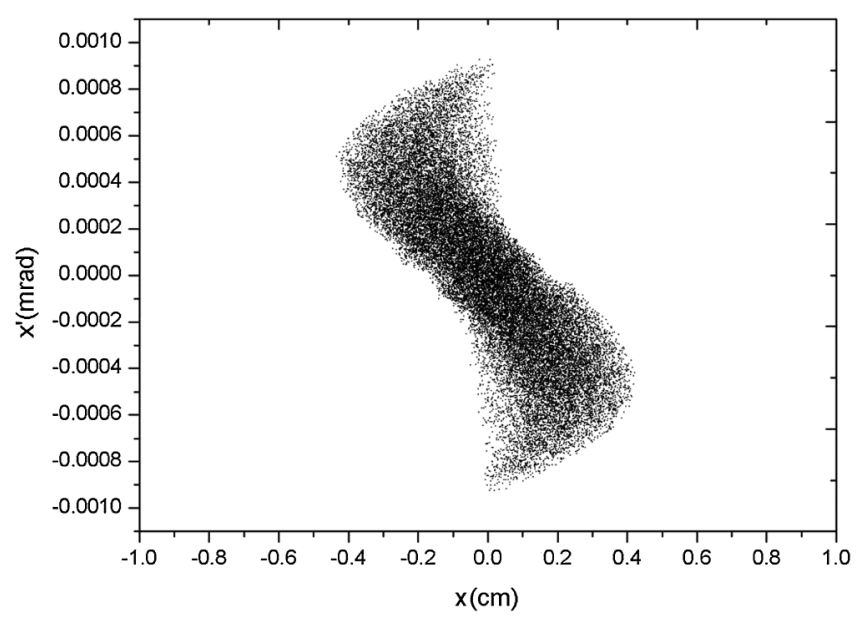

FIG. 6. Particle distribution in the horizontal phase space at the RFQ entrance. 
TABLE II. Main design parameters for the Injector Scheme-I RFQ.

\begin{tabular}{|c|c|}
\hline Parameter & Value \\
\hline rf frequency $(\mathrm{MHz})$ & 325 \\
\hline Injection energy $(\mathrm{keV})$ & 35 \\
\hline Output energy (MeV) & 3.213 \\
\hline Pulsed beam current (mA) & 15 \\
\hline Beam duty factor & $100 \%$ \\
\hline Intervane voltage $V(\mathrm{kV})$ & 55 \\
\hline Beam transmission & $98.7 \%$ \\
\hline Average bore radius $r_{0}(\mathrm{~mm})$ & 2.775 \\
\hline Vane-tip curvature $\rho_{t}(\mathrm{~mm})$ & 2.775 \\
\hline$\rho_{t} / r_{0}$ & 1.0 \\
\hline Maximum surface field (MV/m) & 28.88 (1.62 Kilpatrick field) \\
\hline Cavity power dissipation (kW) & $272.94(1.4 \times$ SUPERFISH $)$ \\
\hline Total power $(\mathrm{kW})$ & 320.94 \\
\hline Average copper power/length $(\mathrm{kW} / \mathrm{m})^{\mathrm{a}}$ & 41.68 \\
\hline Average copper power/area $\left(\mathrm{W} / \mathrm{cm}^{2}\right)^{\mathrm{a}}$ & 3.25 \\
\hline Maximum copper power/area $\left(\mathrm{W} / \mathrm{cm}^{2}\right)^{\mathrm{a}}$ & 3.77 \\
\hline Input normalized rms emittance $(x / y / z)(\pi \mathrm{mm} \operatorname{mrad})$ & $0.2 / 0.2 / 0$ \\
\hline Output normalized rms emittance $(x / y / z)(\pi \mathrm{mm} \mathrm{mrad} /(\pi \mathrm{MeV} \operatorname{deg}))$ & $0.2 / 0.2 / 0.0612$ \\
\hline Vane length $(\mathrm{cm})$ & 467.75 \\
\hline Gap1 (entrance) (cm) & 1.10 \\
\hline Gap2 (exit) (cm) & 1.10 \\
\hline Accelerator length $(\mathrm{cm})$ & 469.95 \\
\hline
\end{tabular}

${ }^{\mathrm{a}}$ The values were obtained by two-dimensional code SUPERFISH [40].

the machine capability in industry domestically based on the construction experience of the previous RFQ (or "973" RFQ: $3.5 \mathrm{MeV}, 352 \mathrm{MHz}, 7 \%$ duty factor) [38] built at IHEP several years ago. (3) Higher beam transmission is favored. Since the lost beam power in a cw RFQ will worsen the deformation of the cavity and vice versa, the deformation of RFQ will decrease the beam transmission further. (4) The rf power dissipation per unit length and per area should be designed as low as possible. This is important for designing the water-cooling system and decreasing the deformation of the RFQ cavity. (5) A modestly low longitudinal emittance at the RFQ exit is beneficial to the dynamics design of the acceleration sections downstream. (6) Redundant water-cooling capability and water-cooling control protection system safeguard the operation of the RFQ. (7) Conservative rf coupler design lowers the coupler's load and ensures the coupler's reliability. Four sets of rf couplers will be adopted and the power load for each set is less than $60 \mathrm{~kW}$. (8) Take full use of the successful experience of the previous RFQ in designing, machining, and operation at IHEP. It is highly time saving and reliable to adopt a similar RFQ structure as the previous one thanks to the similar working frequency and output energy for the two RFQs.

The standard RFQ code chain CURLI, RFQUICK, PARI, and PARMTEQM [39] developed at LANL has been used to design the RFQ. With these codes, the RFQ are physically divided into radial matching section (RMS), shaper, gentle buncher and accelerator four different sections. In order to obtain a good beam transmission and a better bunching, both the shaping and the bunching energy are chosen to be comparatively lower values. In Table II, the main design parameters of the RFQ are listed.

Both the vane-tip curvature $\rho_{t}$ and the average bore radius $r_{0}$ are kept constant $(2.775 \mathrm{~mm})$ along the RFQ with a ratio of 1.0 between them, which are the same as the $973 \mathrm{RFQ}$. A constant vane-tip curvature $\rho_{t}$ is beneficial to the machining of vanes by a formed cutter, and therefore it is both time saving and cost saving in machining, while a constant average bore radius $r_{0}$ benefits the machining of the RFQ cavity and the tuning of field distribution. The design of equal vane-tip curvature and average bore radius benefits the beam transmission of the RFQ, but in the meantime it introduces higher multipole field components, higher surface field, and more rf power dissipations. It is very critical to lower the rf power dissipation per unit length and per area for a RFQ running in $\mathrm{cw}$ mode. Because the rf power dissipation is proportional to the square of the intervane voltage, it is a good way to choose a relatively low intervane voltage of $55 \mathrm{kV}$ and the average rf power dissipation per meter obtained by SUPERFISH is about $41.68 \mathrm{~kW}$, which is close to the rf dissipation power level for a usual heavy-ion RFQ with a lower rf frequency, though the maximum power density is much higher here due to high rf frequency, but it is still within the capability of the cooling system, even taking into account the beam losses which happened at lower energy. Such a low intervane voltage is possible here thanks to the relatively low 


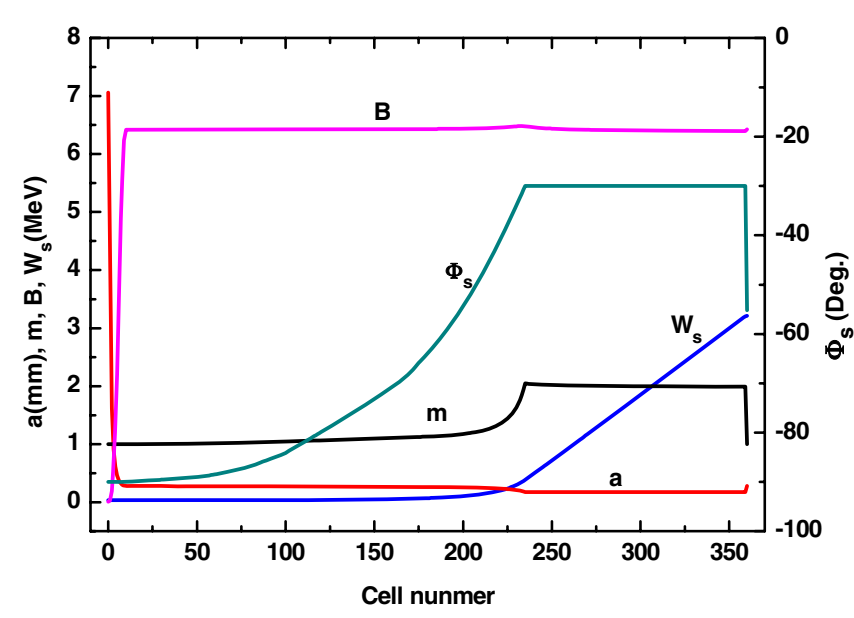

FIG. 7. Main parameter variation with cell number, where $a$ stands for the minimum bore radius, $m$ modulation factor, $B$ focusing factor, $W$ synchronous energy, and $\Phi_{s}$ synchronous phase.

beam current so that the transverse focusing is less demanding. A high beam transmission (about 98.7\%) can be still obtained by using a small average bore radius $(2.775 \mathrm{~mm})$. In Fig. 7 , the main design parameters along cells are shown. In Fig. 8, the phase distributions at the exit of RFQ are plotted.

\section{MEBT1 design}

According to the design of the RFQ, the beam energy will be $3.2 \mathrm{MeV}$ at the RFQ exit. With a beam current of $10 \mathrm{~mA}$, this beam energy in the MEBT1 is quite low so that the space-charge forces have an important impact on the beam dynamics. In addition, the coupling between the transverse and longitudinal planes, rf defocusing, and other causes will also affect the beam dynamics. In order to minimize the emittance growth and halo development along the beam line besides meeting the matching requirements in the phase spaces at the entrance of the superconducting section, the lattice optics should provide strong and uniform focusing. This can be achieved by choosing three sets of quadrupole doublets and a pair of bunchers with more-or-less balanced parameters. A relatively small beam envelope at the bunchers is preferred to ease the technical difficulties of the bunchers. After several iterations between the engineering design and physics design, the layout of the MEBT1 is shown in Fig. 9, and the main parameters of the MEBT1 elements are given in Table III. On the other hand, the MEBT1 line should provide enough beam diagnostics for beam commissioning and tuning, thus sufficient space has to be reserved for installing the diagnostic devices as shown in Fig. 9.

Multiparticle simulations show that both the rms emittance growth and the halo emittance growth are under control in the MEBT1 [4], e.g., less than 5\% for the rms emittance growth in all three phase planes. Some information is also shown in the end-to-end simulations in Sec. III G.

\section{Superconducting section in the injector}

It is one of the most difficult problems in designing the C-ADS injector to quickly and efficiently accelerate the beam from the low energy at the RFQ exit to higher energy while maintaining beam quality at the same time. We have studied and compared several different schemes based on different acceleration structures and lattice structures, including normal conducting cross-bar H-type structure [41], 162.5 MHz HWR structure [42], and $325 \mathrm{MHz}$ low-beta superconducting spoke cavity [43]. For Injector Scheme-I, low-beta spoke cavities in $325 \mathrm{MHz}$ were chosen to accelerate the beam from the $325-\mathrm{MHz}$ RFQ to $10 \mathrm{MeV}$.

Although very low-beta single-spoke cavities are considered very difficult to develop and lack both prototype test and operation experience [44,45], it is straightforward for IHEP to adopt such a spoke cavity for the superconducting acceleration structure in the injector since the main
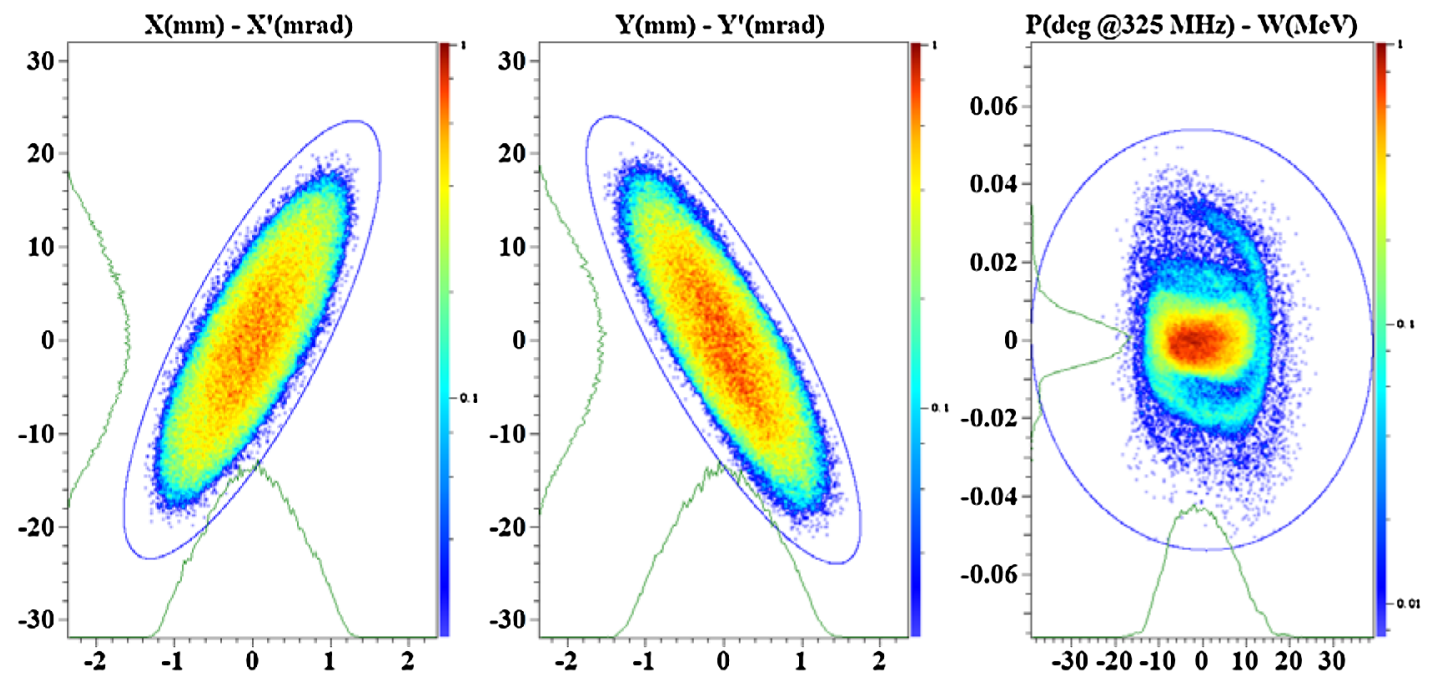

FIG. 8. Phase distributions at the exit of RFQ for the Injector Scheme-I (plotted with TRACEWIN [24]). 


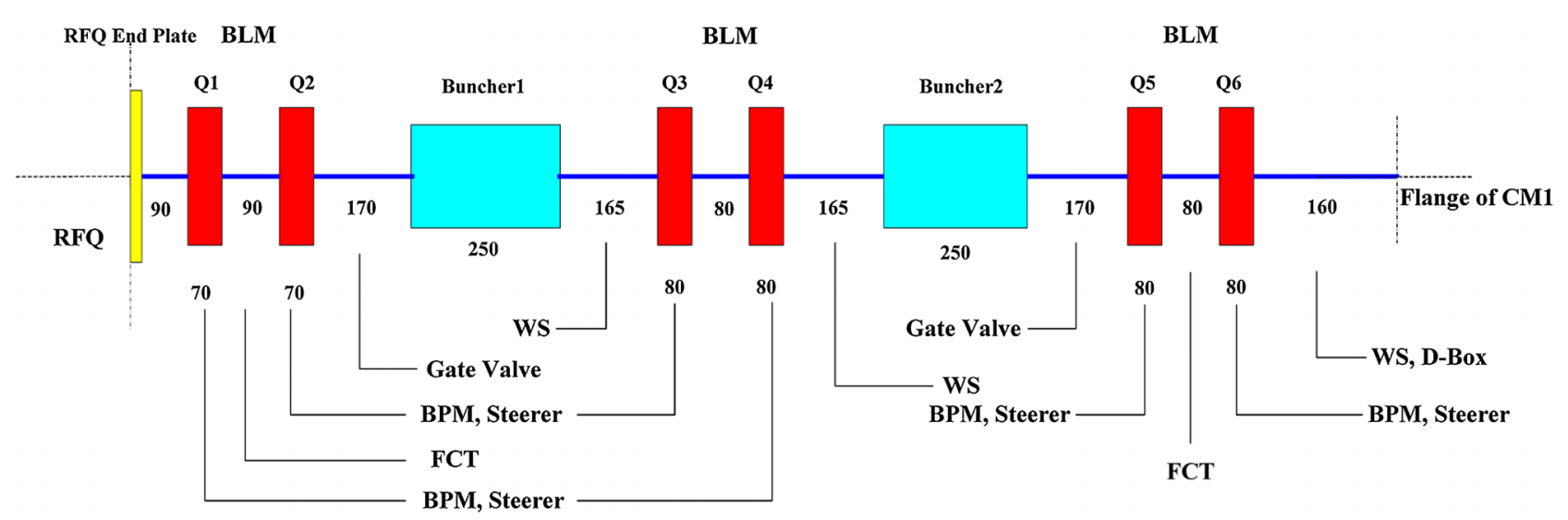

FIG. 9. Schematic of the MEBT1 for Injector Scheme-I.

linac uses the same type of cavities but with higher betas, which will reduce the time and cost in the cavity development. The main design parameters of the cavity are listed in Table IV.

The optimized geometry of the cavity is shown in Fig. 10. The total length of the cavity is $180 \mathrm{~mm}$ and the aperture is $35 \mathrm{~mm}$. The fabrication of the first two prototype cavities has been completed and the vertical test was successfully performed at the end of 2012 [46].

One of the most important principles in high current linac design is to keep the zero-current phase advances per period in all directions less than 90 degrees [47]. From our studies, the phase advance limit is found even more important for low-energy superconducting machines in which some kind of parametric resonance may be excited because of the violation of the smooth approximation [48]. The transverse one can be controlled by properly setting the focusing gradients of the transverse focusing elements,

TABLE III. Main parameters of the MEBT1 elements.

\begin{tabular}{lccc}
\hline \hline Element & $\begin{array}{c}\text { Effective length } \\
(\mathrm{mm})\end{array}$ & $\begin{array}{c}\text { Bore radius } \\
(\mathrm{mm})\end{array}$ & $\begin{array}{c}\text { Field gradient } \\
(\mathrm{T} / \mathrm{m}) / \text { effective } \\
\text { voltage }(\mathrm{kV})\end{array}$ \\
\hline Q1 & 70 & 17.5 & 27.13 \\
Q2 & 70 & 17.5 & -21.08 \\
Q3 & 80 & 27.5 & 11.91 \\
Q4 & 80 & 27.5 & -11.19 \\
Q5 & 80 & 27.5 & 11.25 \\
Q6 & 80 & 27.5 & -10.67 \\
Buncher-1 & 250 & -17.0 & 48.12 \\
Buncher-2 & 250 & -17.0 & 96.07 \\
\hline \hline
\end{tabular}

TABLE IV. Main design parameters of the Spoke012 cavity for the injector.

\begin{tabular}{lccccc}
\hline \hline$\beta_{g}$ & $\begin{array}{c}\text { Frequency } \\
(\mathrm{MHz})\end{array}$ & $\begin{array}{c}U_{\mathrm{acc}} \mathrm{Max} \\
(\mathrm{MV})\end{array}$ & $\begin{array}{c}E_{\max } \\
(\mathrm{MV} / \mathrm{m})\end{array}$ & $\begin{array}{c}B_{\max } \\
(\mathrm{mT})\end{array}$ & $\begin{array}{c}R / Q \\
(\Omega)\end{array}$ \\
\hline 0.12 & 325 & 0.82 & 32.5 & 47.5 & 148.7 \\
\hline \hline
\end{tabular}

while the longitudinal one is directly related to the period length, synchronous phase, and acceleration gradient. The period length is determined by the lengths of the cavity, the transverse focusing elements and the diagnostic devices and the spaces necessary for assembling, and the shorter the better. As discussed in Sec. IIE, superconducting solenoids have more advantages in focusing the low-energy and round beam: more compact and easy to be adapted with the cryogenic environment. Together with the rf defocusing, the transverse focusing structure of SR is determined, where $\mathrm{S}$ denotes solenoid and $\mathrm{R}$ for cavity. The element lengths and the spaces for assembling are determined based on the engineering designs of the cavity, solenoid, BPM, and the cryostat which refer to the designs of similar projects such as Project-X [43] and FRIB [49], and kept as short as possible [50]. Figure 11 shows the element arrangement of a focusing period of Spoke012 section.

With the cavity design parameters listed in Table IV, we need 12 cavities at least to accelerate the beam from 3.2 to $10 \mathrm{MeV}$. Usually it is preferred to have shorter cryomodules housing a modest number of cavities and solenoids for

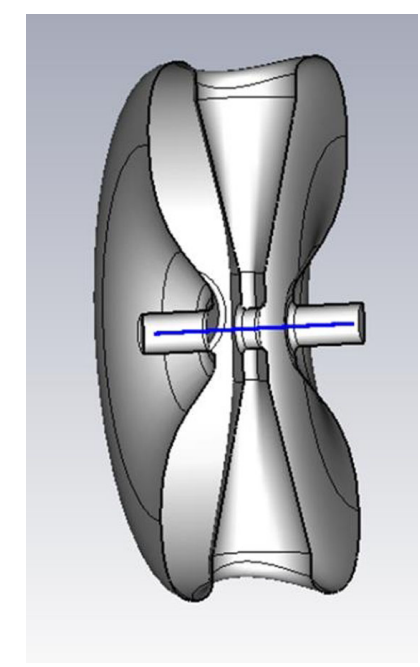

FIG. 10. Optimized geometry of the Spoke012 cavity. 


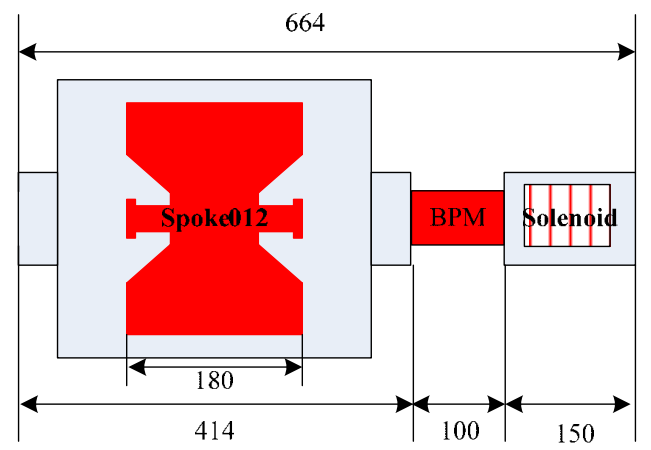

FIG. 11. Layout of one focusing period in Injector Scheme-I.

easier fabrication of cryomodules and easier assembling and alignment of the inside elements. For example, an alternate scheme with two cryomodules for the injector has also been studied but abandoned, where each cryomodule has a length of about 6 meters and houses six cavities. The transition space between two cryomodules will inevitably destroy the periodicity of the focusing array, and the beam quality will be degraded severely. This is especially true when a warm transition is used, as it demands more space. After proper optimization, for example, the length of the cryomodule can be reduced to about $8.5 \mathrm{~m}$. The elements layout in the cryomodule is shown in Fig. 12. In order to reduce the length of the cryomodule, no solenoids are used in both ends of the cryomodule. The beam will be matched to acceleration structures downstream and upstream by the two beam transport lines, MEBT1 and MEBT2.

\section{E. MEBT2 design}

As mentioned before, the C-ADS linac requires two injectors with each of them as the hot spare of the other. It is a real challenge to design a merging beam line to connect the two injectors to the main linac, as this is a highintensity beam requiring very strict control over beam loss and emittance growth. At the low beam energy of $10 \mathrm{MeV}$ and high intensity of $10 \mathrm{~mA}$, it is found difficult to control the emittance growths in the three phase planes when a bending section is present. Several rf bunchers have to be applied to keep the bunch width not too wide to be outside the linear region of the rf field, and the bunchers within the bending section will introduce complexity in the achromatism matching. MEBT2 is also supposed to have a good collimation system to scrape the beam halo and nontrapped particles generated in the injectors in both the transverse and longitudinal planes. Some of the halo particles in the longitudinal phase plane can be removed together with the halo particles in the horizontal phase plane by the collimators in the dispersive region [51]. For each injector, the beam will be directed to the main linac through the main line of the MEBT2 when it is turned into the online operation mode, and the beam will be directed to a beam dump through an auxiliary beam line with a much reduced beam power when it is in the offline mode. The dump line is also used for the commissioning or beam setup of the injector with the full beam power of $100 \mathrm{~kW}$. The layout of a preliminary design is shown in Fig. 13.

For each of the mainstream lines of the MEBT2, eight quadrupoles, two $20^{\circ}$-bending magnets, and four $325-\mathrm{MHz}$ single-gap normal conducting reentrant-type bunchers with maximum voltage of $145 \mathrm{kV}$ are used. In the common part, three quadrupoles, two Spoke012 cavities, and two solenoids are used for the matching in the phase spaces, while another two Spoke012 cavities serve as the backups. A minimum separation of $2.4 \mathrm{~m}$ in the transverse space between the two injectors requires a bending section for each branch was imposed to guarantee the installation space of the two parallel injectors. The design scheme satisfies the requirements of more-or-less uniform transverse focusing, achromatic bending, and good control in the beam phase width. The details of the MEBT2 design can be found in [51,52]. Further optimization for MEBT2 is still under way.

\section{F. Main linac design}

\section{Cavities and lattice structures}

In order to satisfy the rigorous demands on the accelerator stability and reliability, over-design, redundancy, and fault tolerance strategies are implemented in the basic design. The fault-tolerant design in the main linac is guaranteed by means of the local compensation and rematch method [18], which is effective only for a linac composed of short independently powered cavities. To cover the whole energy range from $10 \mathrm{MeV}$ to $1.5 \mathrm{GeV}$ in the main linac, at least four types of superconducting cavities are needed. After optimization, we have chosen two singlespoke cavities working at $325 \mathrm{MHz}$ with geometry $\beta$ of 0.21 and 0.40 , respectively, and two 5-cell elliptical cavities working at $650 \mathrm{MHz}$ with geometry $\beta$ of 0.63 and 0.82 , respectively. The acceleration efficiencies of the four

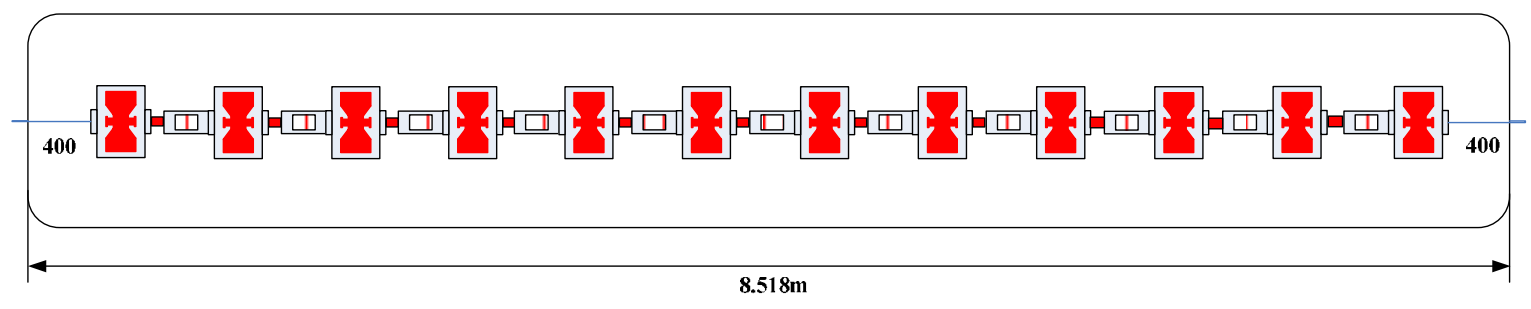

FIG. 12. Schematic of the Spoke012 lattice with one cryomodule. 


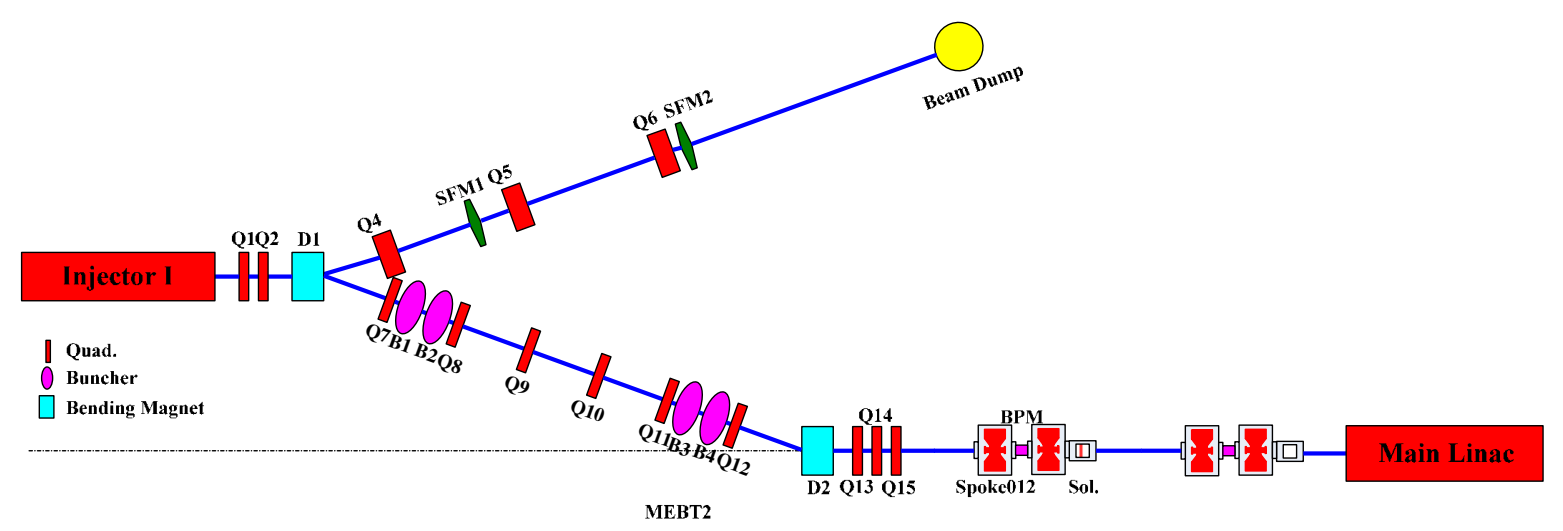

FIG. 13. Schematic of the MEBT2 (only the upper part of two symmetric branches is shown).

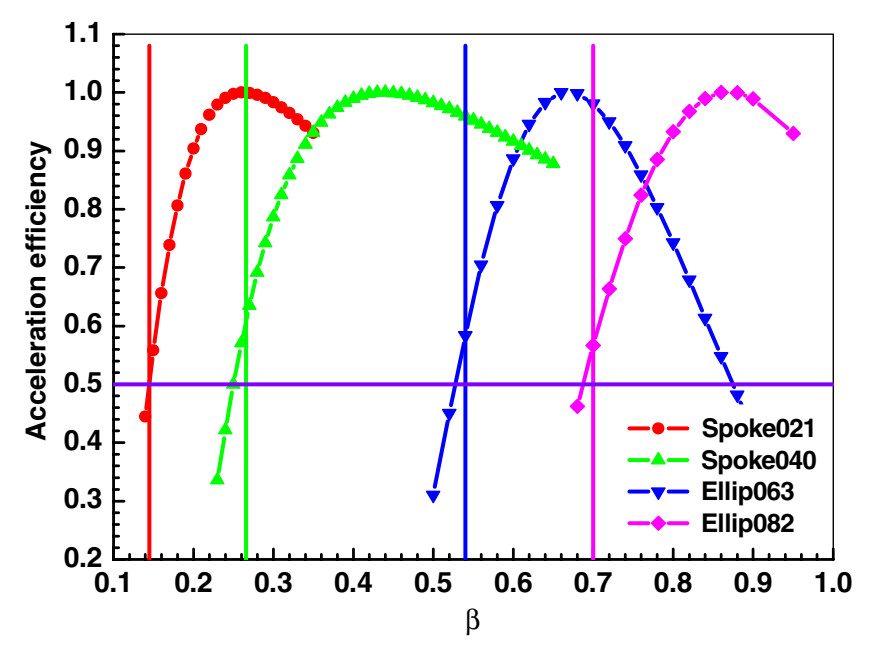

FIG. 14. Acceleration efficiency curves of the SC cavities in the main linac.

cavities and their effective energy ranges are shown in Fig. 14. The effective energy ranges for the four types of cavities are all shifted to the lower energy to fit the special phase advance law required by the stable beam dynamics. The main design parameters of the cavities are listed in Table V. For the nominal design, only $3 / 4$ of the maximum cavity voltage is used, whereas another $1 / 4$ is reserved for the local compensation, and this redundancy is also beneficial to the cavity reliability. The variation in performance for practical cavities coming from the fabrication process can also be included in the reserved capacity.

There are four sections each consisting of one type of cavity in the main linac, Spoke021 and Spoke040 for the lower-energy part and Ellip063 and Ellip082 for the higherenergy part. The lattice structures for each section are shown in Fig. 15, and they are characterized by long drifts in both sides of each period. With these kinds of lattice structures, the cryomodules are more flexible. Each cryomodule can accommodate one lattice period, two periods, or even more periods without affecting the beam dynamics performance and is totally decided by the mechanical and engineer considerations. Furthermore, it also helps in minimizing the possibility of mismatch within one section. The matching between two sections is guaranteed by varying the parameters of the adjacent cavities and transverse focusing elements. With warm transitions between cryomodules, the replacement of failure cryomodules can be easily carried out; in addition, beam diagnostics and collimators can be arranged in the warm sections.

In total, thirty-eight Spoke021 cavities in seven cryomodules, sixty-four Spoke040 cavities in eight cryomodules, forty-two Ellip063 cavities in 14 cryomodules, and 100 Ellip082 cavities in 20 cryomodules are used in the main linac, where the two first Spoke021 cavities and the four Spoke012 cavities in the MEBT2 are housed in a special cryomodule. All the cryomodules have modest length of 5-10 m.

\section{Beam dynamics design}

The phase advances per cell in the three phase planes are usually kept below $90^{\circ}$ to avoid the parametric resonance $[47,48,53]$. Except in the matching cells at the section transitions and in the Ellip063 section, the focusing fields in both the transverse and longitudinal directions are kept almost constant in each section to have almost constant envelope amplitude when the geometrical rms emittance is shrinking along the acceleration [54]. This also means constant synchronous phase in each section, but the absolute value of the synchronous phase decreases from the

TABLE V. Main parameters of the SC cavities in the main linac.

\begin{tabular}{lccrcc}
\hline \hline $\begin{array}{l}\text { Cavity } \\
\text { type }\end{array}$ & $\beta g$ & $\begin{array}{c}\text { Frequency } \\
(\mathrm{MHz})\end{array}$ & $\begin{array}{c}V_{\max } \\
(\mathrm{MV})\end{array}$ & $\begin{array}{c}E_{\max } \\
(\mathrm{MV} / \mathrm{m})\end{array}$ & $B_{\max }(\mathrm{mT})$ \\
\hline Spoke021 & 0.21 & 325 & 1.64 & 31.14 & 65 \\
Spoke040 & 0.40 & 325 & 2.86 & 32.06 & 65 \\
Ellip063 & 0.63 & 650 & 10.26 & 37.72 & 65 \\
Ellip082 & 0.82 & 650 & 15.63 & 35.80 & 65 \\
\hline \hline
\end{tabular}



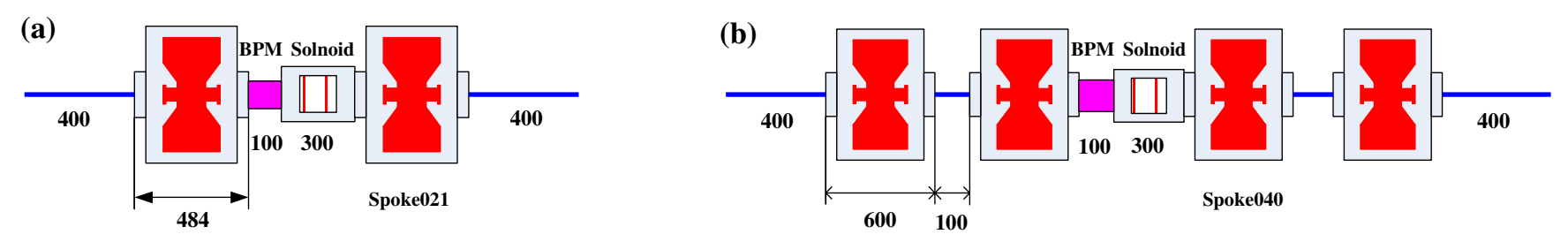

(c)
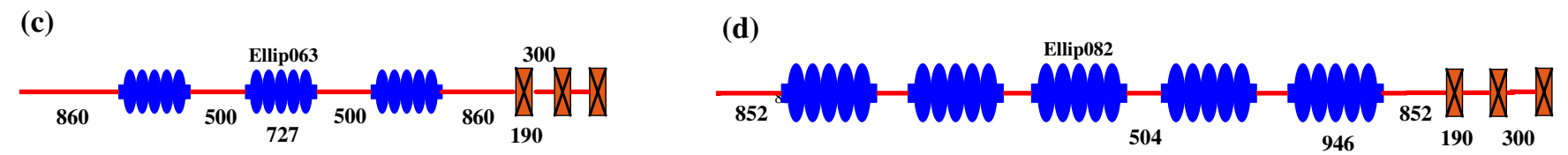

FIG. 15. Schematic view of the lattice structures for the main linac sections: (a) for Spoke021, (b) for Spoke040, (c) for Ellip063, (d) for Ellip082.

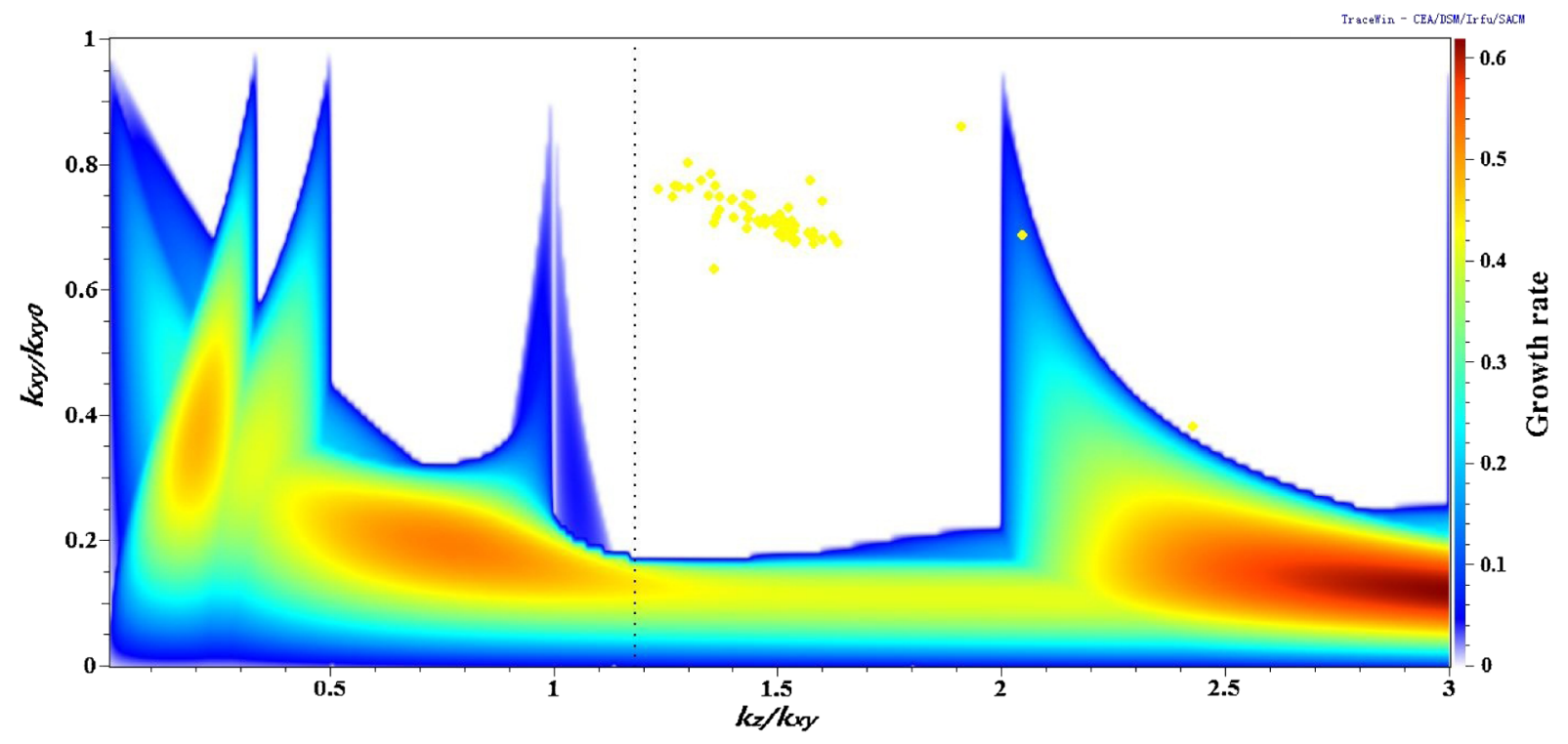

FIG. 16. Tune footprint in the Hofmann chart for the main linac. Growth rates are in units of zero space-charge betatron tune.

lower-energy section to the higher-energy section to obtain higher acceleration rate while maintaining the acceptance and $6 \mathrm{D}$ water-bag emittance ratio larger than 10 , a low beam-loss design criteria for the acceptance in the main linac. Because of the limitation in the longitudinal phase advance per cell, the cavity voltages at the beginning parts of the sections may not be fully exploited.

Since the phase advance per cell should be below $90^{\circ}$, it is better to set the longitudinal phase advance larger than the transverse one to obtain higher acceleration efficiency. This design must be considered together with the equipartition condition if we want to avoid the energy transfer between different freedoms [55]. When the longitudinal emittance is smaller than the transverse one, it is easy to set up the working point at the resonance-free region in the Hofmann chart. This explains why the RFQ design was optimized to have a relatively small longitudinal emittance.

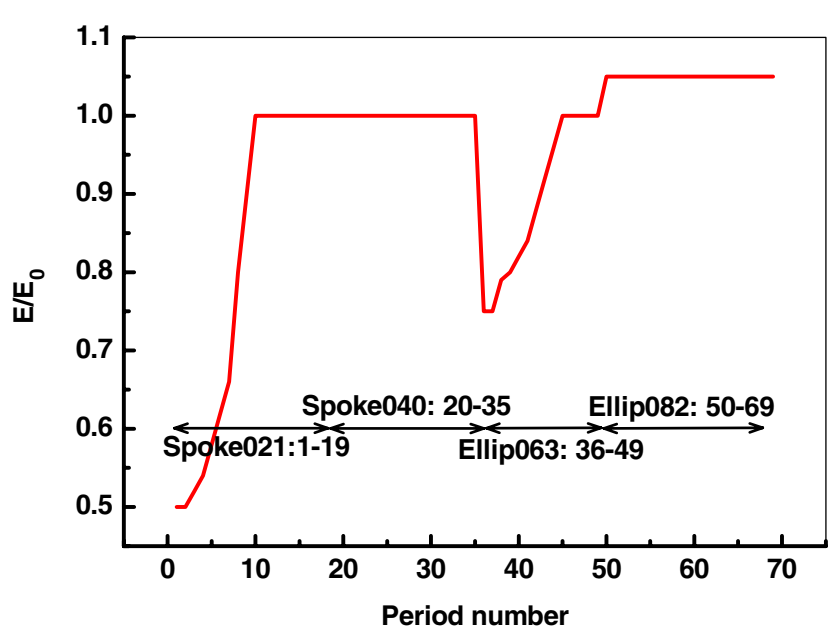

FIG. 17. Effective rf voltages in use for all the cavities as compared with the nominal voltages. 


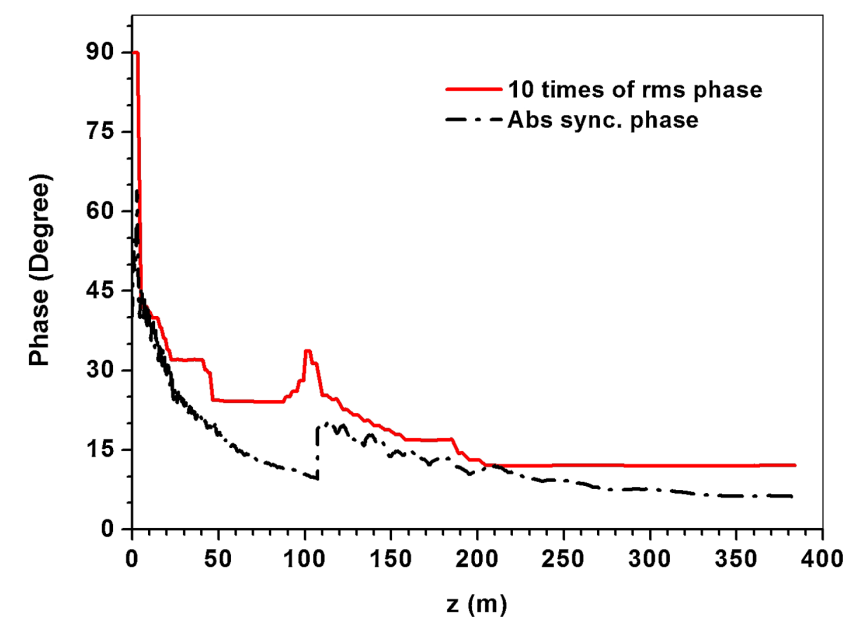

FIG. 18. Absolute synchronous phase and 10 times RMS phase width along main linac.

Although there are three transitions for the four sections of different lattice structures and cavity types, the one between the section Spoke040 and the section Ellip063 is the most important. One reason is the rf frequency doubling from Spoke040 to Ellip063, and the other is the
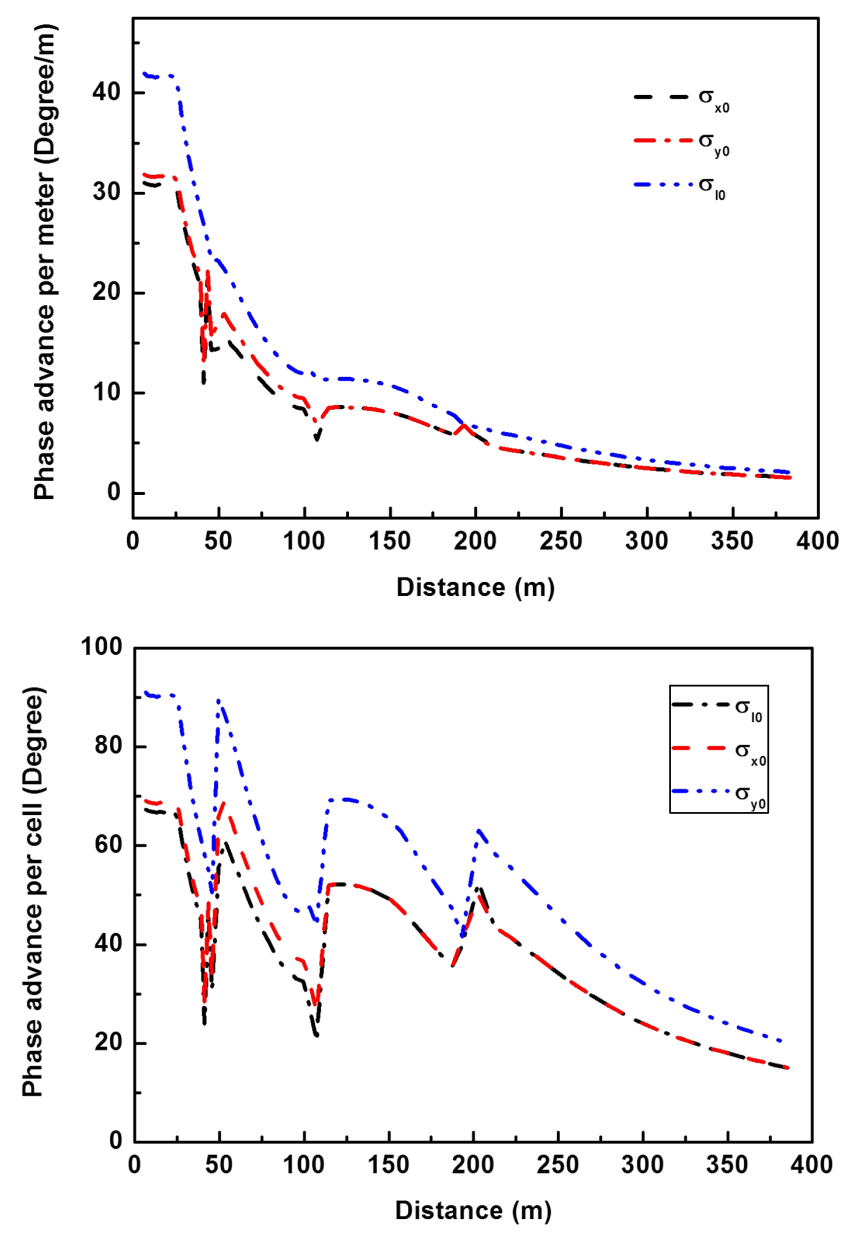

FIG. 19. Phase advances per meter (upper) and phase advances per cell (lower) along the main linac.

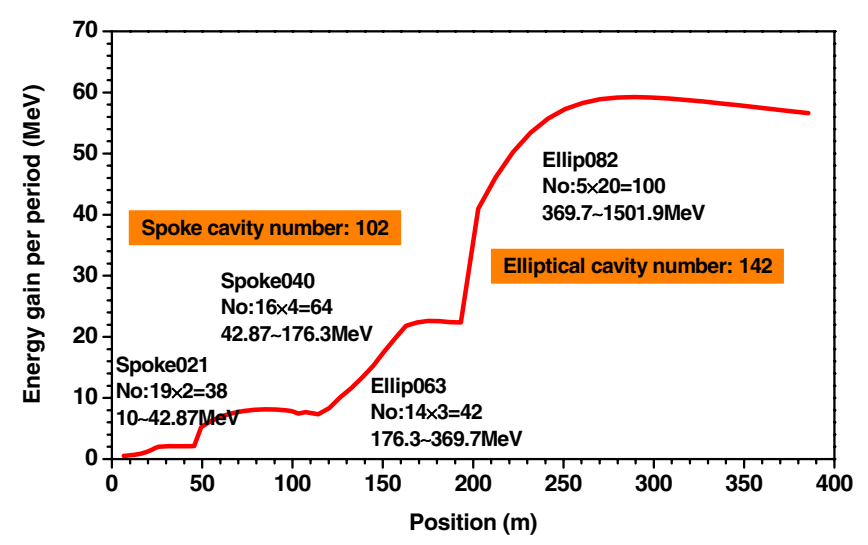

FIG. 20. Energy gain per meter in the main linac.

transverse focusing from inside-cryomodule solenoids to room-temperature quadrupole triplets. Careful matching should be carried out to avoid important emittance growth at the transitions. One important thing is to make smooth changes in focusing, and another is to avoid the absolute synchronous phase being too small.

Different phase advance ratios between the longitudinal and the transverse planes have been studied. It turns out that the phase advance ratio of 0.75 is adopted after the compromise among the equipartition condition, the acceptance to emittance ratio, and the phase advances per cell. Figure 16 shows the tune footprint in the Hofmann chart. We can see that, except for one point falling in the very weak part of the $k_{z} / k_{x}=2$ resonance region, all the other points are in the resonance-free region.

Figure 17 shows the effective rf voltage in use as compared with the nominal voltage for all four types of superconducting cavities. It is the optimized result by following the requirements on the phase advance, smooth change in focusing, and longitudinal acceptance. Another limitation on the effective field level comes from the multipacting effect in the superconducting spoke cavities. The voltage ratio should not be lower than 0.5 to avoid the possibility of

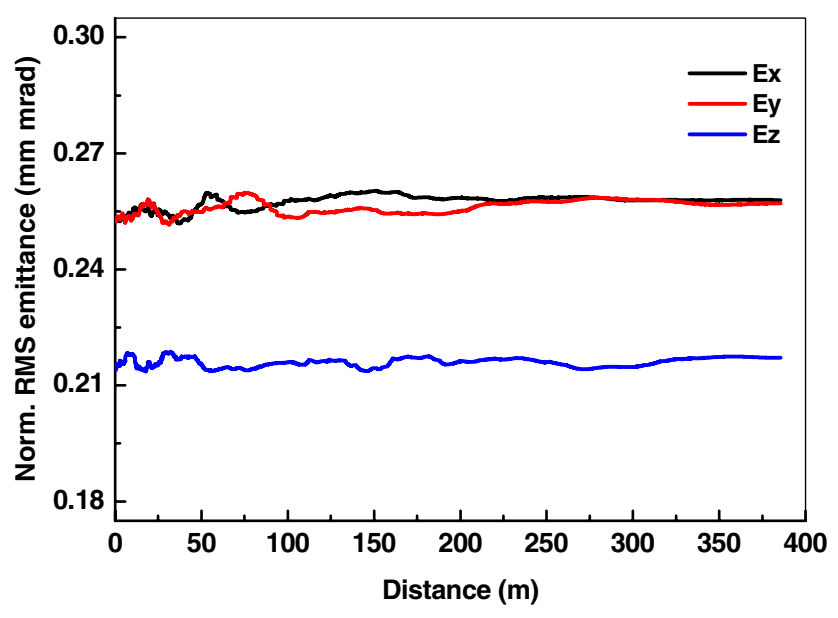

FIG. 21. Evolution of the emittance along the main linac. 

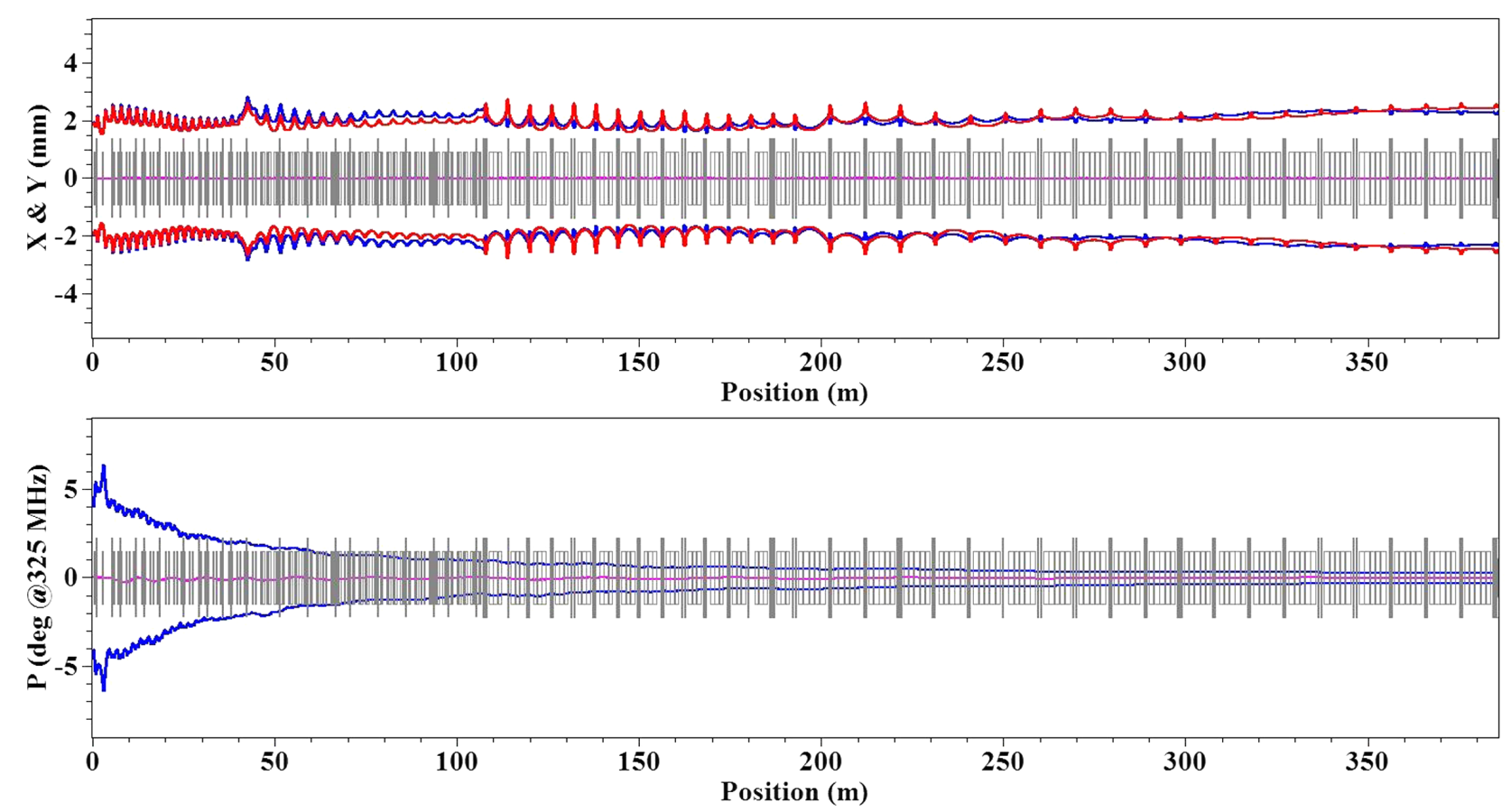

FIG. 22. Root-mean square envelope evolutions in the main linac (upper: $X$ in red, $Y$ in blue; lower: phase width).

working in the multipacting regions. To reduce beam loss, the synchronous phase is kept larger than 10 times the RMS phase width throughout the main linac as shown in Fig. 18. The phase advances per meter and phase advances per cell are shown in Fig. 19, and they change quite smoothly. Figure 20 shows the energy gain per meter along the linac.

Detailed multiparticle simulations have been performed for the main linac. As the first step, using an initial 6D water-bag distribution we have studied the dynamic behavior of the beam core or the properties in $\mathrm{rms}$ along each of the major sections, then combined them to form the main linac. The rms emittances are $0.24 \pi \mathrm{mm}$ mrad for the transverse planes and $0.216 \pi \mathrm{mm}$ mrad for the longitudinal plane, respectively at the entrance of the main linac, assuming the emittance growths by $20 \%$ in the injector and in the MEBT2 section. It is found that the transverse emittance growths are $3 \%$ and $2.8 \%$ for the horizontal and vertical planes, respectively, and the longitudinal emittance growth is almost nothing $(-0.3 \%)$. The transverse rms beam size in average is about $2.5 \mathrm{~mm}$. The simulation results are shown in Figs. 21-23. From the simulation results, we can find that the rms emittance growth along
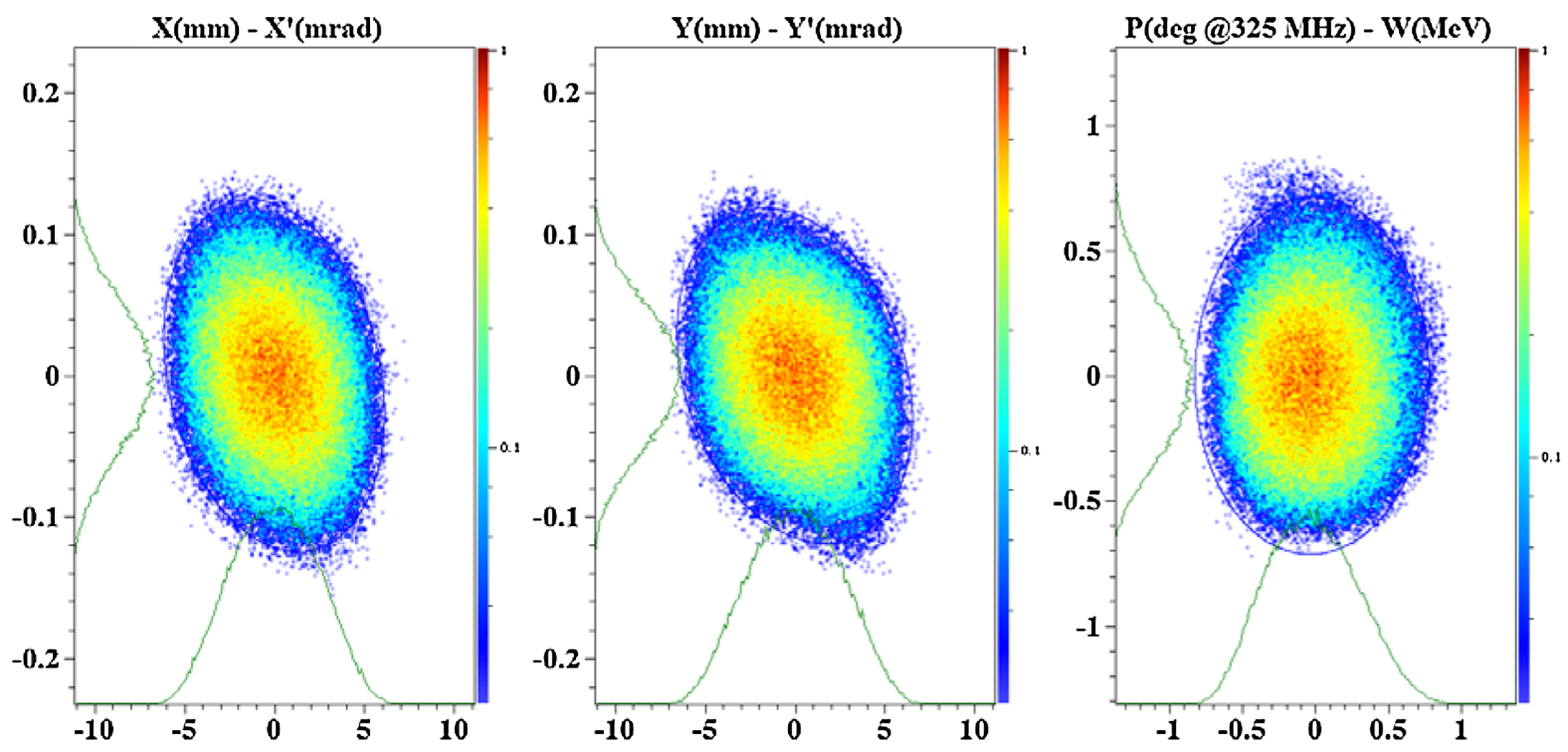

FIG. 23. Beam distributions in the phase spaces at the exit of the main linac (@1.5 GeV). 
the main linac is under control in all three phase spaces, e.g., about a few percent. The envelope evolutions are also smooth along the main linac.

As the halo development due to errors, mismatches and resonances is key in causing beam loss, it becomes the central focus of the beam dynamics studies once the lattice and the basic dynamic behavior are determined using the beam core or the rms emittance. As this is a linac of very high beam power, beam loss should be controlled at the level of $10^{-8} / \mathrm{m}$. This means that the behaviors of very
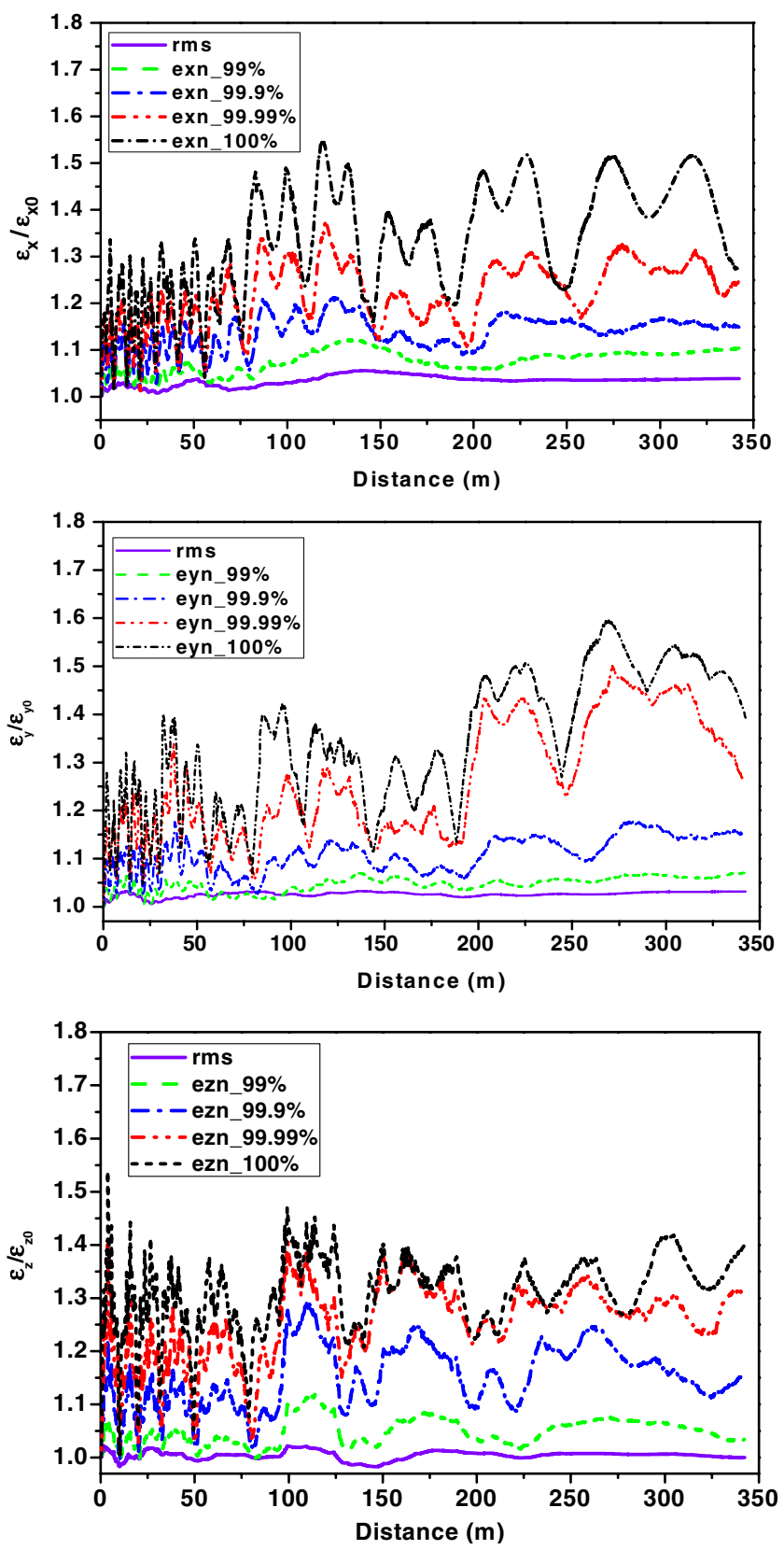

FIG. 24. Halo development along the main linac for different beam fractions. Emittances for different fractions are compared with the ones at the beginning of the main linac $\left(\varepsilon_{z} / \varepsilon_{x}=0.85\right.$, $\left.k_{x} / k_{z}=0.75\right)$. sparse halo particles should be studied. Therefore, the emittance evolutions for $99 \%, 99.9 \%, 99.99 \%$, and $100 \%$ beam fractions have been studied, using both TRACK and TRACEWIN codes. The number of macroparticles is $10^{5}$ for the simulations.

The error related studies will be presented in Sec. V; some mismatches due to component failures are presented in Sec. IV. Here we will show the resonances-related studies. The imperfect matchings at the section transitions are also included as we perform end-to-end simulations for the main linac.

For the halo development related to the space-charge resonances, we have carried out simulations on the halo formation to see if the working point is sensitive or not. Figure 24 shows the emittance evolutions with the nominal working point $\left(\varepsilon_{z} / \varepsilon_{x}=0.85, k_{x} / k_{z}=0.75,10 \mathrm{~mA}\right.$, Injector Scheme-I), which is free from dangerous resonances. Figure 25 shows the longitudinal emittance evolution with the working point situated in the resonance region in the Hoffman chart $\left(\varepsilon_{z} / \varepsilon_{x}=0.85, k_{x} / k_{z}=1.0\right)$. We can find that even for a modest beam current of $10 \mathrm{~mA}$, the resonance plays a significant role in the halo development; therefore, we adopt the design with the working point in resonant-free regions.

\section{G. End-to-end multiparticle simulations}

End-to-end beam dynamics simulations are performed from the input of the RFQ to the end of the linac to verify the matching between each part. It was also recommended that the end-to-end simulations should be started from the plasma surface of the ion source since the initial particle distribution is very important to the beam properties in the downstream, and this can be pursued in the future. The initial particle distribution at the entrance of the RFQ is assumed as 4D water-bag distribution with transverse rms emittance of $0.2 \pi \mathrm{mm}$ mrad and the total particle numbers are 100000 , and the optimized distributions at

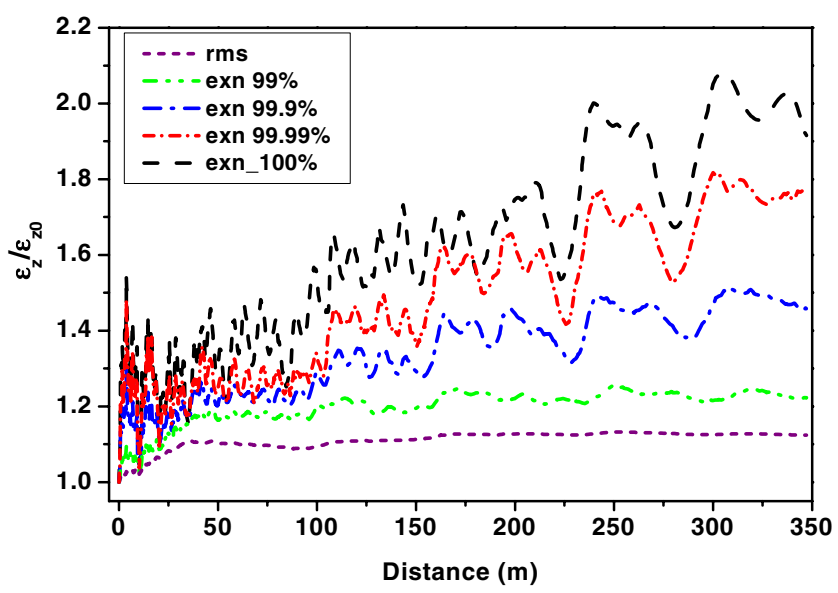

FIG. 25. Longitudinal emittance development along the main linac for different beam fractions for the working point situated at the resonance $\left(k_{x} / k_{z}=1.0\right)$. 


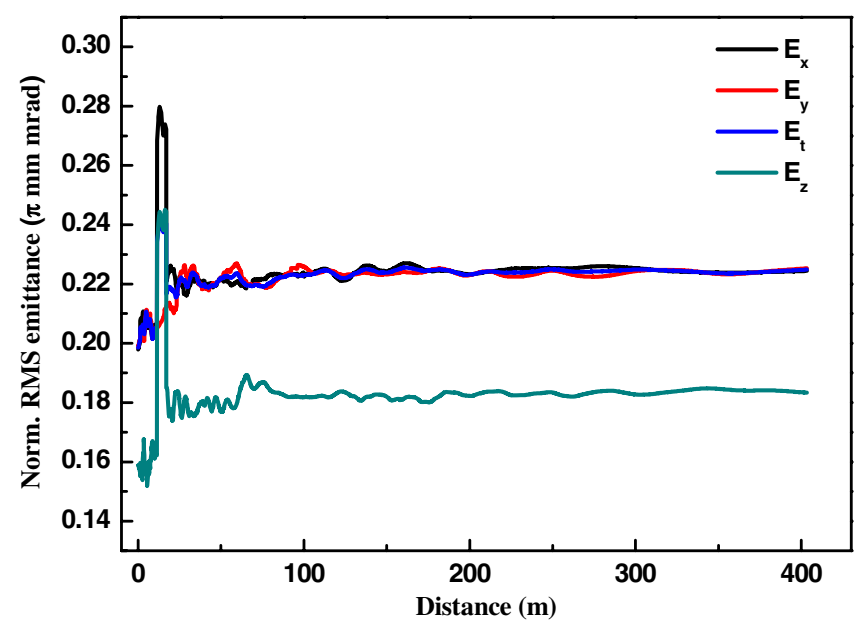

FIG. 26. Evolutions of the normalized rms emittances along the linac.

the RFQ exit are applied as the initial particle distributions for TRACEWIN. In the following, only the simulation results after the RFQ are given.

Figure 26 shows the evolutions of normalized rms emittances along the linac. Both the transverse and longitudinal emittances are nearly constant along the main linac part, but about 20\% emittance growth in the longitudinal plane and $10 \%$ in the transverse planes can be observed in MEBT2, just as expected. It indicates that the matching and design for the core beam along the linac are good; the smooth rms envelopes shown in Fig. 27 also indicate this.
Besides the rms emittances, the evolutions of halo emittance including different fractional particles are also studied. As shown in Fig. 28, the MEBT2 contributes to very important growth of the $100 \%$ emittance, almost 5 times in the transverse planes. It is expected that some halo particles can be collimated in MEBT2 with proper collimation schemes. The phase space distributions at the end of the linac are shown in Fig. 29.

\section{COMPENSATION AND REMATCH SCHEMES FOR MAJOR ELEMENT FAILURES}

In order to achieve the required reliability for the C-ADS accelerators, besides all the hardware which is operated with conservative performance and redundancy, it is still important to have fault-tolerant capabilities in the design $[1,17]$. For example, modular solid-state rf powers are very useful in increasing their reliability. However, no matter how we improve the hardware's reliability performance, it should be expected to meet some failures of important devices but with a much lower rate. The accelerator design has to deal with these situations. In the following, we will discuss how to compensate the failures of two kinds of major components: superconducting resonators and transverse focusing elements (solenoids and quadrupoles).

\section{A. SC cavity failures}

Cavity failures are considered to be one of the most important hardware failures [10,56]. Several factors may cause cavity failures: rf power source, coupler, low level
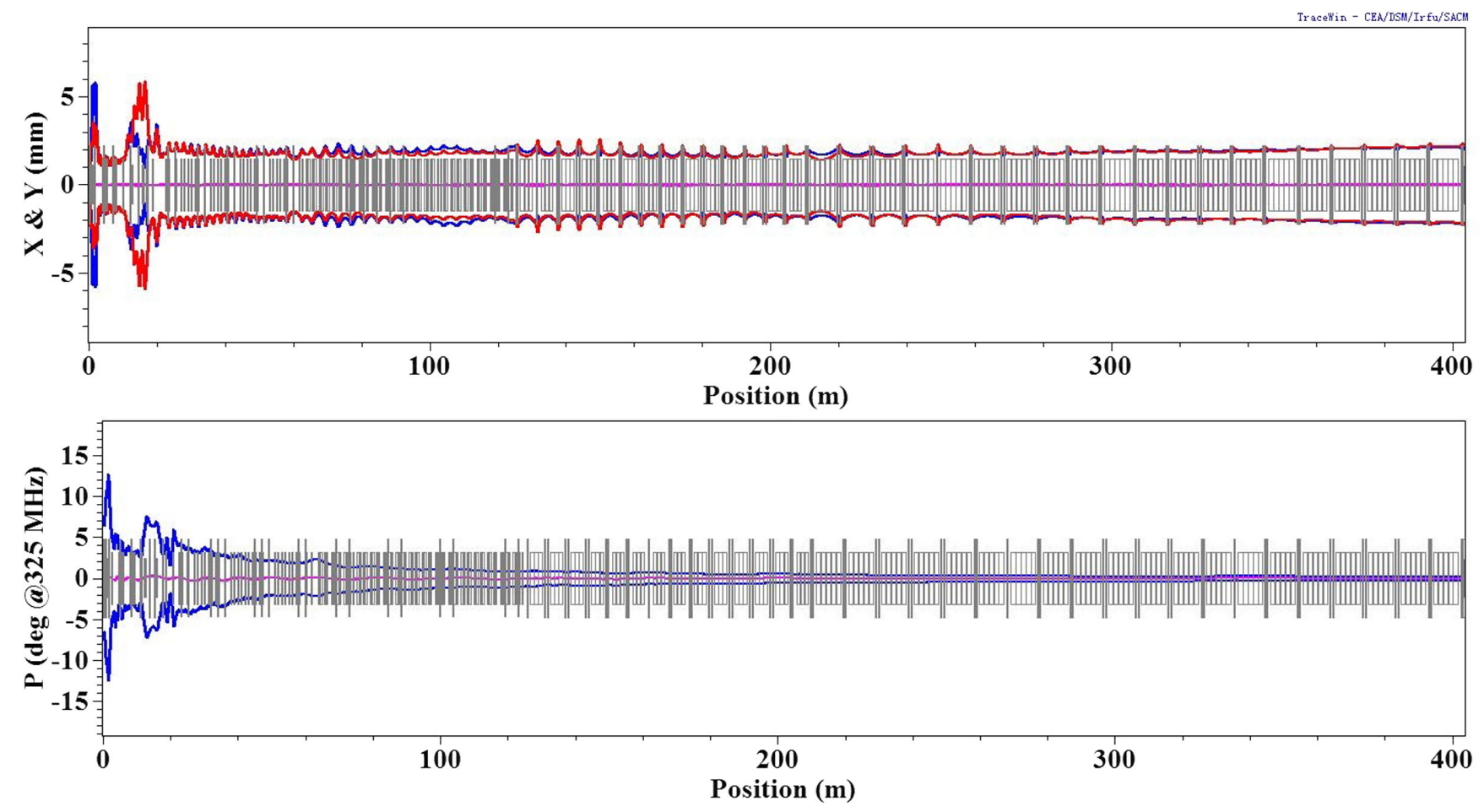

FIG. 27. Root-mean square envelope evolutions in the linac (upper: $X$ in red, $Y$ in blue; lower: phase width). 

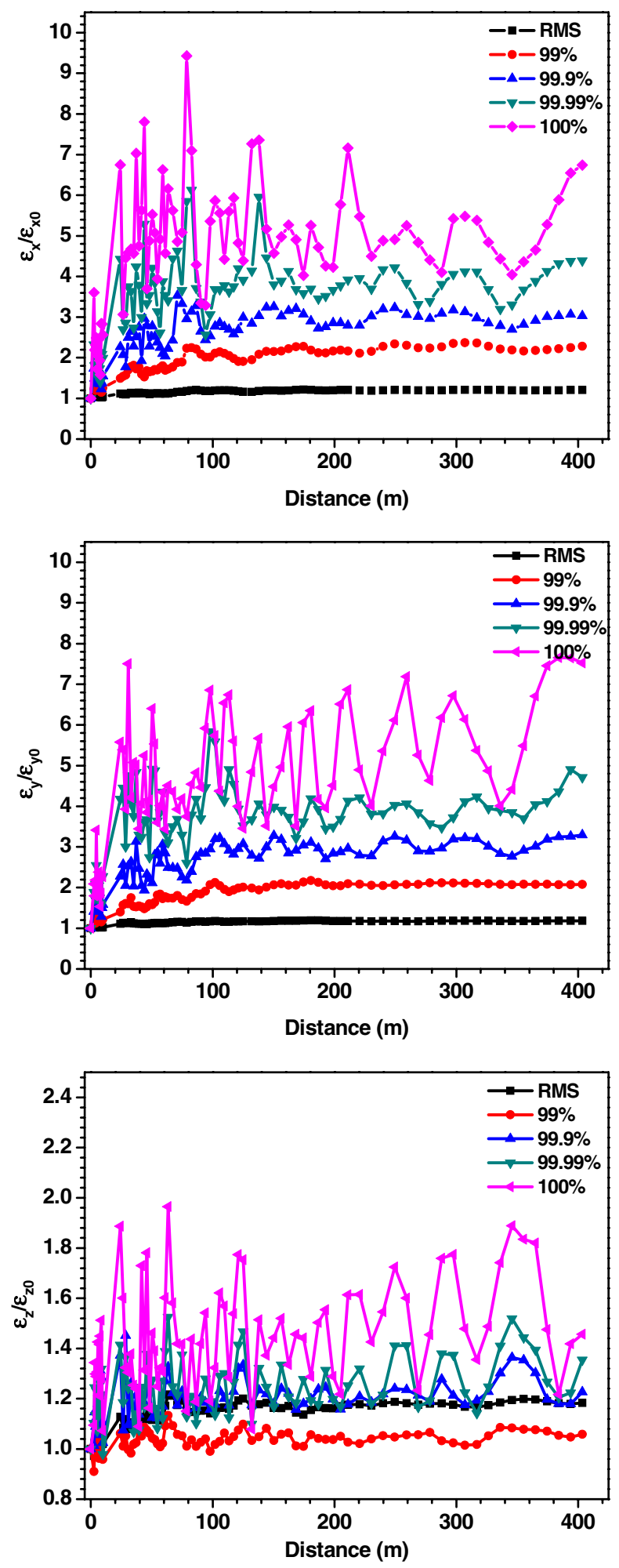

FIG. 28. Halo development along the linac for different beam fractions.

radio frequency system, cavity mechanical tuning, etc. When a cavity fails, if nothing is done, the whole or part of the beam will be lost in the downstream linac [18]. The reason is that the phase slip caused by the velocity change will make the beam center phase to exceed the longitudinal acceptance of the downstream acceleration section. The best way to deal with this kind of failure is to readjust the settings of the neighboring cavities to regain the nominal velocity, and to rematch the transverse focusing at the same time as the rf cavities also affect the transverse focusing. At SNS, it is the usual operation to adjust all the downstream SC cavities when one fails (or "global compensation method" [57]). However, it takes some minutes to make the adjustment and the beam should be cut off during the tuning. For C-ADS, the beam stop time should be controlled within a few seconds and it is better to make the compensation by just involving only a few neighboring cavities so that more cavity failures in different locations can be compensated independently and efficiently [10]. It is also found that at lower energy the local compensation method is much more efficient in controlling the emittance growth than the global compensation method.

The local compensation method for cavity failures has been successfully applied in all the subsections of the main linac $[4,58]$. Almost no growth in the rms emittance and slight growth of a few percent in halo emittance have been obtained. Taking the example of a failure of one cavity in the middle part of the Spoke040 section, Fig. 30 shows the local compensation method. As mentioned earlier, the nominal peak surface electric field in operation is also derated from the applicable 32.5 to $25 \mathrm{MV} / \mathrm{m}$, so that about one-fourth reserve can be used for the compensation and rematch. The multiparticle simulations show no significant growths in the rms emittances and the halo emittances after applying the local compensation and rematch method.

\section{B. Focusing element failures}

From the operation experience of the existing accelerator, the failures of the transverse focusing elements such as quadrupoles and solenoids are much less frequent than $\mathrm{rf}$ cavities; however, they are still very important in an ADS linac due to the very strict requirement on reliability. Rematch is also needed to minimize the emittance blowup due to the failures. Major failures come from quench in SC magnets or solenoids, power supplies, and control units. Because of quite different focusing structures, the rematches for SC solenoid failures and quadrupole failures are also very different.

For solenoid failures, we found that the rematch is much more difficult, especially in the low-energy sections. As we adopted the long period lattice and there is only one solenoid in one cell for transverse focusing, once the solenoid fails, the beam size in the cell or neighboring cells will become significantly larger even if the beam is rematched by the neighboring solenoids. Large transverse beam size will result in the emittance distortion due to the nonlinear rf fields and space-charge force. If other elements in the cell could provide additional transverse 

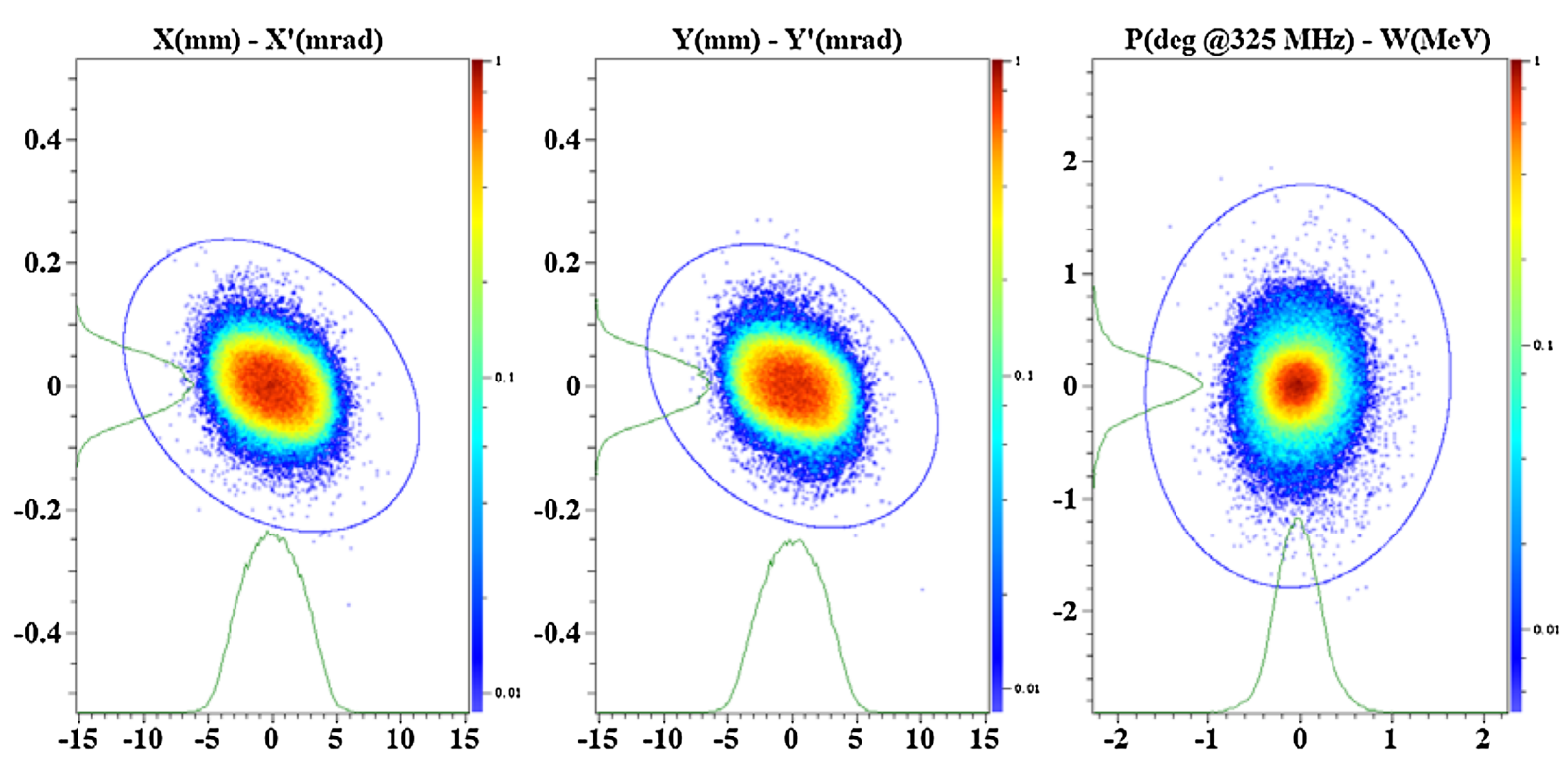

FIG. 29. Phase space distributions at the exit of linac (@1.5 GeV).

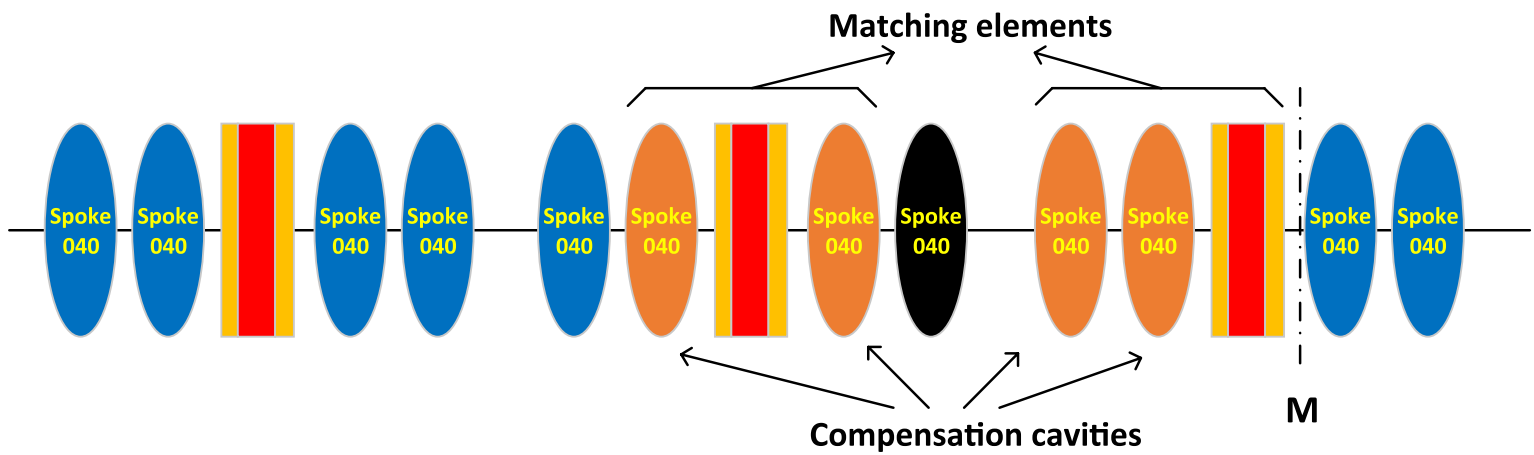

FIG. 30. Local compensation for a cavity failure in the middle Spoke040 section. (The blue ellipses stand for cavities, the red squares for solenoids, the black ellipse for the failed cavity, and the orange ellipses for major compensation cavities. M stands for matching point.)

focusing while the solenoid fails, the beam halo growth could be controlled. Then we studied the possibility of inversing the synchronous phase of one of the cavities in the cell to provide additional transverse focusing, namely we change the synchronous phase from negative to positive when keeping the acceleration. The nominal transverse focusing structure RSR or DFD will become FD or DF in the rematch mode. This method has been proven successful. As focusing element failures are more critical in the cells with large phase advances, namely in the beginning part of each spoke sections, we take the failure of the solenoid in the middle of the Spoke021 section as an example, as shown in Fig. 31. The synchronous phase of the first cavity in the same cell is inversed from $-33^{\circ}$ to $40.5^{\circ}$, the neighboring cavities and solenoids are adjusted to achieve the local rematch goal at the matching point. Multiparticle simulations show less than $10 \%$ growth in the rms emittances and about 30\% growth in the halo emittance growth after applying the local compensation and rematch method.
The rematch method for the failures of the quadrupole magnets in the Ellip063 and Ellip082 sections has also been studied. It is also due to the rematch for failures that we changed the transverse lattice from the earlier doublet-based structure to the triplet-based structure. The triplet-cell focusing structure is considered to have advantage over doublet-cell structure: when one of the three quadrupole fails, the two others can be easily transformed into a doublet to rematch the rms beam at the matching point with little mismatching. Slight readjustments in the two neighboring triplets can help to make almost a perfect rematch including beam halo. Unlike in the case of solenoid failures, there is no need for putting the neighboring cavities in the rematch.

There are also other possible failures in the main linac that affect the normal operation of the accelerator, such as failures of cryomodules, beam diagnostics devices, computer network, vacuum system, etc. Cryomodule failures are fatal so that the accelerator cannot continue to operate, and the beam has to shut down when they are repaired or replaced by 


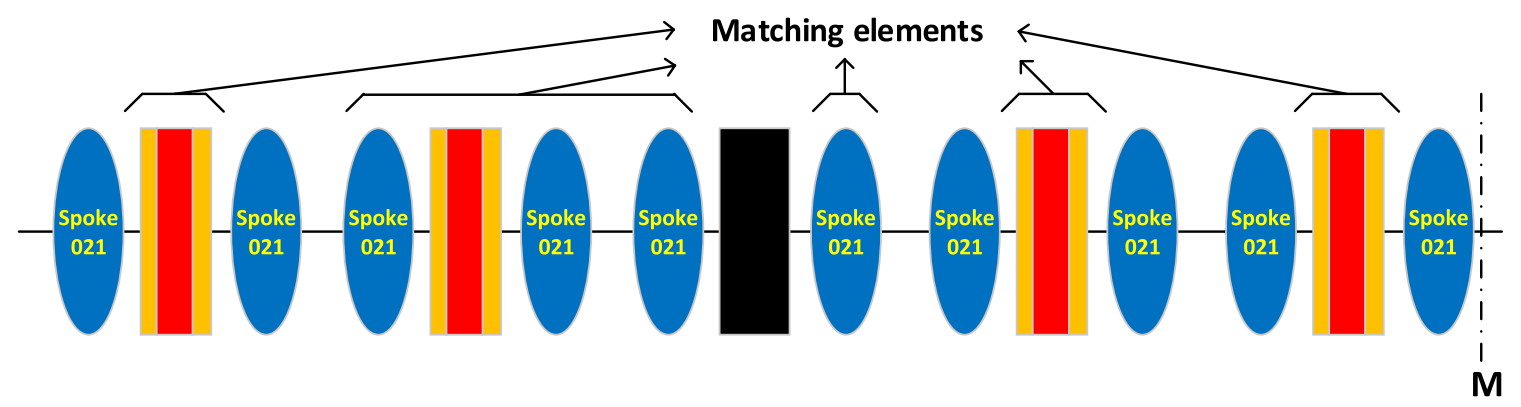

FIG. 31. Local rematch method for the failure of the SC solenoid in the middle of the Spoke021 section.

a new one. Some beam diagnostic devices failures and vacuum pump failures can be tolerated. The computer network may need backup to guarantee normal operation.

\section{ERROR AND BEAM-LOSS STUDIES}

\section{A. General considerations}

Although all the magnets installed in the beam lines should be aligned strictly with special alignment technique, the residual errors of the installation, field imperfections, and variations give rise to important beam off-centering. The off-centering may lead to direct beam loss due to the aperture limitation or significant emittance growth due to mismatching in the phase space that will probably result in beam loss in the downstream sections. The beam off-centering from most of the static errors can be corrected by a proper correction scheme that employs a number of correction magnets together with some beam position monitors which are also aligned strictly in the beam lines. The correctors are often integrated into SC solenoids and quadrupoles to save the precious longitudinal space. Therefore, the beam can be centered to the optical axis within an acceptable tolerance. However, even with orbit corrections and rematches the beam quality will still degrade mainly due to the nonlinearity of rf field and space-charge force. The halo

TABLE VI. Amplitudes of static errors used for error studies.

\begin{tabular}{lcccc}
\hline \hline $\begin{array}{l}\text { Element } \\
\text { errors }\end{array}$ & $\begin{array}{c}\text { Solenoid } \\
\text { Alignment/field } \\
\text { error }\end{array}$ & $\begin{array}{c}\text { Spoke cavity } \\
\text { Alignment/rf } \\
\text { error }\end{array}$ & $\begin{array}{c}\text { Elliptical cavity } \\
\text { Alignment/rf } \\
\text { error }\end{array}$ & $\begin{array}{c}\text { Quadrupole } \\
\text { Alignment/field } \\
\text { error }\end{array}$ \\
\hline$\Delta x(\mathrm{~mm})$ & \pm 1 & \pm 1 & \pm 1 & \pm 0.1 \\
$\Delta y(\mathrm{~mm})$ & \pm 1 & \pm 1 & \pm 1 & \pm 0.1 \\
$\Delta z(\mathrm{~mm})$ & \pm 1 & \pm 1 & \pm 1 & \pm 0.5 \\
$\phi x(\mathrm{mrad})$ & \pm 2 & \pm 2 & \pm 2 & \pm 2 \\
$\phi y(\mathrm{mrad})$ & \pm 2 & \pm 2 & \pm 2 & \pm 2 \\
$\phi z(\mathrm{mrad})$ & & \pm 2 & & \pm 0.5 \\
$\Delta E(\%) / \Delta B(\%)$ & \pm 0.5 & \pm 1 & \pm 1 & \\
$\phi \mathrm{RF}\left({ }^{\circ}\right)$ & & \pm 1 & & $\pm 0.1 \mathrm{~mm}$ \\
$\mathrm{BPM}$ accuracy & & & & \\
\hline \hline
\end{tabular}

${ }^{\mathrm{a} B P M}$ accuracy: measurement accuracy and misalignment (offset).

TABLE VII. Amplitudes of dynamic errors used for error studies

\begin{tabular}{lcccc}
\hline \hline $\begin{array}{l}\text { Element } \\
\text { errors }\end{array}$ & $\begin{array}{c}\text { Solenoid } \\
\text { Alignment/field } \\
\text { error }\end{array}$ & $\begin{array}{c}\text { Spoke cavity } \\
\text { Alignment/rf } \\
\text { error }\end{array}$ & $\begin{array}{c}\text { Elliptical cavity } \\
\text { Alignment/rf } \\
\text { error }\end{array}$ & $\begin{array}{c}\text { Quadrupole } \\
\text { Alignment/field } \\
\text { error }\end{array}$ \\
\hline$\Delta x(\mu \mathrm{m})$ & \pm 10 & \pm 10 & \pm 10 & \pm 2 \\
$\Delta y(\mu \mathrm{m})$ & \pm 10 & \pm 10 & \pm 10 & \pm 2 \\
$\Delta z(\mu \mathrm{m})$ & \pm 2 & \pm 2 & \pm 2 & \pm 2 \\
$\phi x(\mathrm{mrad})$ & \pm 0.02 & \pm 0.02 & \pm 0.02 & \pm 0.02 \\
$\phi y(\mathrm{mrad})$ & \pm 0.02 & \pm 0.02 & \pm 0.02 & \pm 0.02 \\
$\phi z(\mathrm{mrad})$ & & \pm 0.02 & & \pm 0.02 \\
$\Delta E(\%) / \Delta B(\%)$ & \pm 0.05 & \pm 0.5 & \pm 0.5 & \pm 0.05 \\
$\phi \mathrm{RF}\left({ }^{\circ}\right)$ & & \pm 0.5 & \pm 0.5 & \\
\hline \hline
\end{tabular}



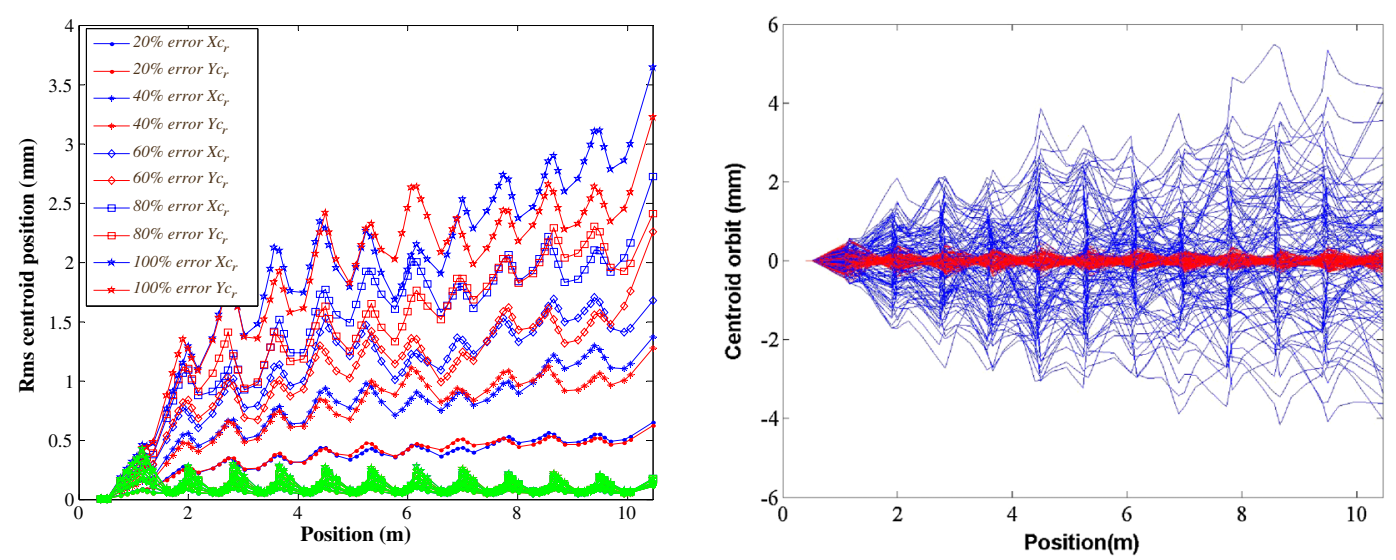

FIG. 32. Simulation results with different schemes in the Spoke012 section (left: rms centroid errors with different alignment error strength, green for after correction; right: centroid trajectories, blue for before correction, red for after correction).

formation is the most important source of beam loss which is critical in very high beam power accelerators, such as the C-ADS linac.

On the other hand, the beam off-centering and mismatches from dynamic field and alignment errors cannot be corrected, due to their random property in time. Therefore, they will result in direct emittance growth or halo formation and should be controlled by limiting the stabilities or ripples of the power supplies and the rf power sources.

The changes in the Twiss parameters due to the static errors can also be corrected by rematching with the measured beam profile data; if not corrected, the mismatches in envelope will lead to emittance growth. However, this correction is only effective to large errors as profile monitor measurements are not precise. Small mismatches cannot be detected by the profile monitors and they are included in the dynamics simulations. As many as possible beam profiles are planned to be installed in the warm sections between cryomodules.

\section{B. Influence of different errors}

There are inevitably installation errors including translational errors and rotation errors, and also field errors [59] for all electromagnetic devices which are used to focus, guide, or accelerate the beams. For focusing magnetic fields, the amplitude rippling coming from power supplies is the main source of dynamics errors, and the frequency spectrum does not need to be considered here; for rf fields, the dynamic errors mainly come from the amplitude and phase fluctuations of the rf power sources and the cavity instabilities. As mentioned above, static errors are usually larger but they can be corrected or compensated to a large extent. On the contrary, most of the dynamic errors are usually smaller with an exception of the dynamic rf errors that are considered almost similar to the static rf errors, but they cannot be corrected. Tables VI and VII show the initial error definitions for the error studies [60-65]. The errors in displacement and rotation are uniformly distributed and the rms value is the maximum divided by $\sqrt{3}$ [60]. The operational experience at the Spallation Neutron Source (SNS) shows that even for superconducting cavities operating in pulsed mode with strong Lorentz force detuning, the $\mathrm{rf}$ errors can be controlled below $0.5^{\circ}$ in phase and $0.5 \%$ in amplitude [66].

It is important to perform beam centering during the beam commissioning or beam setup and also minor adjustment during operation. The beam position monitors (BPMs) measure the beam centroids both in $x$ and $y$ directions. A beam alignment program uses the BPMs output data to set correctors which steer the beam back to the optical axis. However, BPMs have also installation errors and measurement errors [64]. With beam calibration, the BPM errors can be reduced significantly. It is found that the BPM error plays a key role in the orbit correction system [65].

Error studies become more crucial at the C-ADS linac than in other proton linacs, due to three reasons: the first one is the extremely strict requirement on beam loss; the second one is that as a number of rf cavities, focusing solenoids and BPMs are housed in a long cryostat, it is

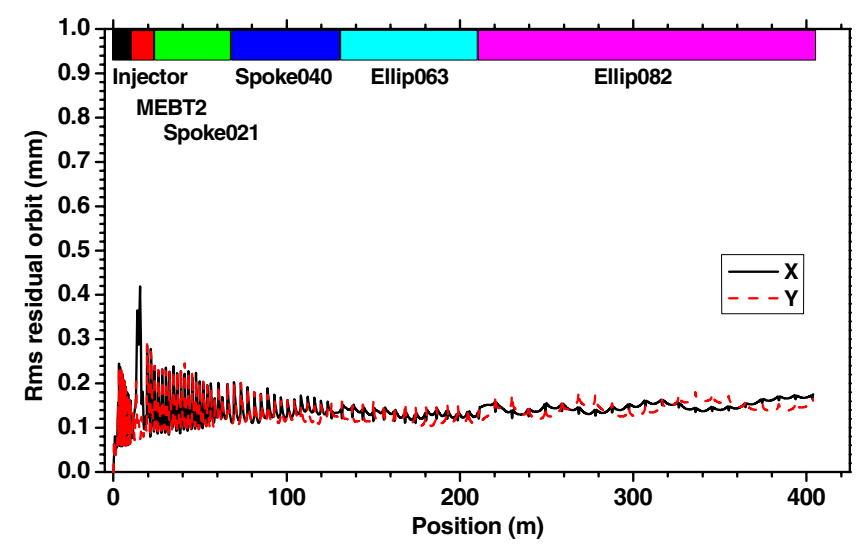

FIG. 33. Root-mean square residual orbit along the linac with errors and correction. 
TABLE VIII. Root-mean square emittance growths and beam loss in the end-to-end simulations with and without errors.

\begin{tabular}{lccccc}
\hline \hline & & & & & Maximum \\
& $\begin{array}{c}E x \\
(\%)\end{array}$ & $\begin{array}{c}E y \\
(\%)\end{array}$ & $\begin{array}{c}E z \\
(\%)\end{array}$ & $\begin{array}{c}\text { Average } \\
\text { loss rate }\end{array}$ & $\begin{array}{c}\text { loss power } \\
(\mathrm{W})\end{array}$ \\
\hline $\begin{array}{l}\text { Without } \\
\text { error }\end{array}$ & 11.8 & 12.0 & 15.3 & 0 & 0 \\
$\begin{array}{l}\text { With error } \\
\text { (meanlrms) }\end{array}$ & 36.110 .9 & 38.512 .7 & 33.711 .9 & $1.6 \times 10^{-7}$ & 3.3 \\
\hline \hline
\end{tabular}

much more difficult to align them in cold temperature than in warm temperature; and the third one is that the phase advances per cell are quite large, thus the error influence becomes more significant.

In the studies of errors and correction schemes, five important factors should be considered: beam-loss rate, rms residual orbit or maximum residual orbit, emittance growth, energy deviation, and corrector strength. Eventually, it is the beam-loss distribution along the linac that has the determinant influence over the design and the operation. Other factors will lead to potential beam loss downstream.

\section{Orbit correction schemes}

The impacts of the misalignments and field errors of the focusing elements and the superconducting cavities as well as a correction scheme for the central orbit have been investigated for all the linac segments. Table VI lists the misalignment tolerances of all beam elements and static rf errors that are used for the orbit correction studies.

According to the lattice design, a pair of BPM and corrector in each period is responsible for the orbit correction. The correctors are attached to the solenoids in the spoke sections, and can be independent magnets in the elliptical sections. Figure 32 shows the orbit errors along the Spoke012 section without correction and with correction

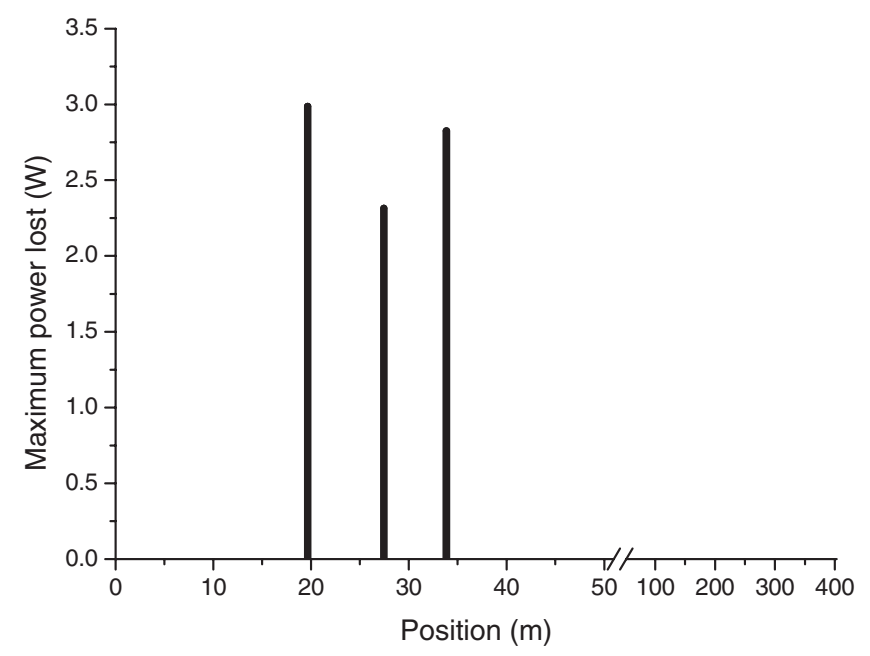

FIG. 34. Averaged power distribution of the lost particles along the linac. including BPM errors. For each set of alignment errors, TRACEWIN or TRACK code is used to minimize the central orbit deviations at the BPM locations using the available correctors. The simulations have also been carried out on the other sections. In all these cases, we can obtain corrected orbit errors (rms) within $0.5 \mathrm{~mm}$, which is considered acceptable. Even after the orbit error correction, the rms emittance growth is still significant, about $10 \%$ compared to the case without errors.

\section{Simulation results with errors}

In order to investigate the performance of the real linac, the end-to-end simulations with errors listed in
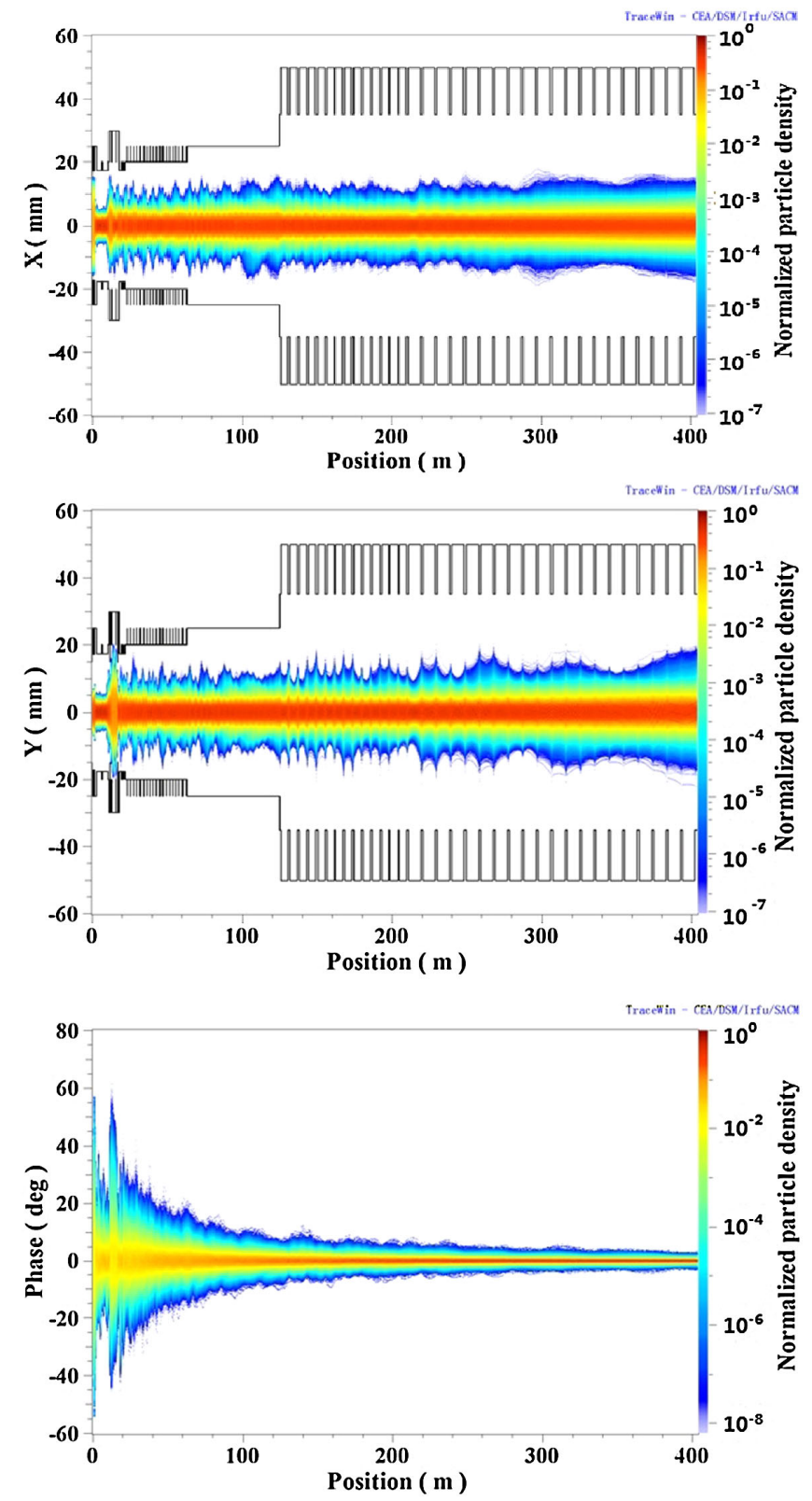

FIG. 35. Particle trajectories in the horizontal plane (upper), vertical plane (middle), and longitudinal plane (lower). 
Tables VI and VII have also been carried out. Starting from the exit of the RFQ, 1000 different linacs were generated by errors with $10^{5}$ particles for each linac. By applying the orbit correction scheme as mentioned in the last section, the residual orbit is less than $0.2 \mathrm{~mm}$ in most of the main linac, even the maximum excursion of $0.4 \mathrm{~mm}$ in MEBT2 is within the predefined upper limit of the residual orbit $(0.5 \mathrm{~mm})$. The maximum strength for the correctors is $1.5 \times 10^{-3} \mathrm{Tm}$. Figure 33 shows the rms residual orbit along the linac. Table VIII lists the rms emittance growth with or without errors. It can be seen that with errors there is about an additional $20 \%$ average emittance growth in the transverse planes and in the longitudinal plane and it causes particle losses of $1.6 \times 10^{-7}$, and the lost particle distribution along the linac is shown in Fig. 34. There are $1.3 \%$ linacs that have lost particles and the maximum loss power is about $3.3 \mathrm{~W}$ which should be collimated at MEBT2. Particle trajectories with all errors in the transverse plane are shown in Fig. 35.

More detailed error studies including collimation are under way.

\section{SUMMARY}

The physics design for the C-ADS accelerators has been carried out based on the state-of-art technology in high-power proton linacs, which shows no stop for the project to be pursued in multiple phases. Although the design work is still preliminary and needs to be proceeded with much more efforts in the future, the actual conceptual design of the C-ADS accelerator physics shows that it can meet the basic requirements on beam dynamics for the $\mathrm{C}$ ADS project. This study also provides the basic schemes and the fundamental parameters for the hardware R\&D and the construction of the test stands.

The main design features of the C-ADS linac include: two parallel injectors with one as the hot spare of the other, optimized cw RFQ design with special attention on heat load and cooling, independently powered superconducting acceleration structures along the linac except the RFQ, sophisticated medium-energy beam transport line to merge two injectors to the main linac, local compensation and rematch for major element failures to meet the very strict reliability requirement, extremely strict control on beamloss level by controlling emittance growth along the linac and localized collimation, etc. Multiparticle simulations so far including space-charge effect and all kinds of errors support the design.

\section{ACKNOWLEDGMENTS}

The authors want to thank all the C-ADS accelerator colleagues at both IHEP and IMP for the support and discussions, and also the international review committee members for many valuable suggestions and comments during and beyond the reviews. The study is supported by the CAS Strategic Priority Research Program-Future
Advanced Nuclear Fission Energy (Accelerator-Driven Subcritical System), and also by National Natural Science Foundation of China (Projects No. 11235012 and No. 10875099).

[1] R.L. Sheffield, in Proceedings of HB2010, Morschach, Switzerland, 2010 (PSI, Viligen, Switzerland, 2011), pp. 1-5.

[2] Y. He et al., in Proceedings of IPAC 2011, San Sebastian, Spain (EPS-AG, Spain, 2011), pp. 2613-2615.

[3] Shuhui Liu, Yuan He, and Zhijun Wang, in Proceedings of HB2012, Beijing, China, 2012.

[4] J. Y. Tang and Z.H. Li, Report No. IHEP-CADS-Report/ 2012-01E.

[5] S. Nagaitsev, in Proceedings of the 2011 Particle Accelerator Conference, NY, USA (IEEE, New York, 2011), p. 2056.

[6] P. N. Ostroumov, New J. Phys. 8, 281 (2006).

[7] J.-L. Biarrotte, F. Bouly, S. Bousson et al., in Proceedings of the 23rd Particle Accelerator Conference, Vancouver, Canada, 2009 (IEEE, Piscataway, NJ, 2009), pp. 668-672.

[8] P. A.P. Nghiem, N. Chauvin, O. Delferriere et al., in Proceedings of HB2010, Morschach, Switzerland, 2010 (Ref. [1]), pp. 309-313.

[9] A. Facco, A. Balabin, R. Paparella et al., in Proceedings of Linac08, 2008, Victoria, BC, Canada, pp. 257-259.

[10] J.-L. Biarrotte, in Proceedings of the 9th European Particle Accelerator Conference, Lucerne, 2004 (EPSAG, Lucerne, 2004), pp. 1282-1284.

[11] G. Ciovati et al., in Proceedings of the 19th Particle Accelerator Conference, Chicago, Illinois, 2001 (IEEE, Piscataway, NJ, 2001), pp. 484-486.

[12] W. Hartung et al., in Proceedings of the 20th Particle Accelerator Conference, Portland, OR, 2003 (IEEE, New York, 2003), pp. 1362-1364.

[13] K. W. Shepard, in Proceedings of the 20th Particle Accelerator Conference, Portland, OR, 2003 (Ref. [12]), pp. 581-585.

[14] J. R. Delayen, in Proceedings of Linac 2004, Luebeck, Germany (2004), pp. 589-593.

[15] A. Facco et al., in Proceedings of the 9th European Particle Accelerator Conference, Lucerne, 2004 (Ref. [10]), pp. 1012-1014.

[16] A. Facco et al., in Proceedings of the 10th European Particle Accelerator Conference, Edinburgh, Scotland, 2006 (EPS-AG, Edinburgh, Scotland, 2006), pp. 448-450.

[17] H. Safa, A. Muller, and B. Carluec, Report No. XADSDEL04-063, 2004.

[18] J.-L. Biarrotte and D. Uriot, Phys. Rev. ST Accel. Beams 11, 072803 (2008).

[19] G. P. Lawrence, in Proceedings of the 19th International Linear Accelerators Conference, Chicago, Illinois, 1998 (NTIS, Springfield, VA, 1998).

[20] J.-L. Biarrotte, in Proceedings of LINAC2004, Luebeck, Germany (2004).

[21] M. Reiser, Theory and Design of Charged Particle Beams (Wiley-VCH Verlag $\mathrm{GmbH} \& \mathrm{Co}$. KGaA, Weinheim, 2008), p. 346.

[22] I. Hofmann, Phys. Rev. E 57, 4713 (1998). 
[23] I. Hofmann and O. Boine-Frankenheim, Phys. Rev. Lett. 87, 034802 (2001).

[24] I. Hofmann, G. Franchetti, O. Boine-Frankenheim, J. Qiang, and R. Ryne, Phys. Rev. ST Accel. Beams 6, 024202 (2003).

[25] S. Bousson et al., in Proceedings of LINAC 2006, Knoxille, Tennessee USA (2006), pp. 704-706.

[26] The Spiral 2 Project APD report, 2005 [http://pro.ganilspiral2.eu/spiral2/what-is-spiral2/apd].

[27] http://laacg1.lanl.gov/laacg/services/download_sf.phtml.

[28] http://irfu.cea.fr/Sacm/logiciels/index3.php.

[29] V.N. Aseev, P. N. Ostroumov, E.S.Lessner, and B. Mustapha, in Proceedings of the 21st Particle Accelerator Conference, Knoxville, 2005, edited by C. Horak (IEEE, Piscataway, NJ, 2005), p. 2053.

[30] Z. M. Zhang et al., in Proceedings of ECRIS'12, Sydney, 2012, FRXA02.

[31] Huachang Liu, Huafu Ouyang, Jun Peng, Tao Huang, Keyun Gong, Taoguang $\mathrm{Xu}$, and Shinian $\mathrm{Fu}$, Nucl. Instrum. Methods Phys. Res., Sect. A 654, 2 (2011).

[32] K. R. Crandall, TRACE 3-D Documentation Report No. LA-11054-MS, Los Alamos, 1987.

[33] Yuri K. Batygin, Nucl. Instrum. Methods Phys. Res., Sect. A 539, 455 (2005).

[34] GPT User Manual, Pulsar Physics [http://www.pulsar.nl/gpt/].

[35] L. M. Young, L.J. Rybarcyk, J.D. Schneider, M.E. Schulze, and H. V. Smith, in Proceedings of the 20th International Linac Conference, Monterey, CA, 2000 (SLAC, Menlo Park, CA, 2000), p. 336.

[36] Fu Shi-Nian, Ouyang $\mathrm{Hua}-\mathrm{Fu}$, and $\mathrm{Xu}$ Tao-Guang, Chinese Phys. C 29, 3 (2005), in Chinese.

[37] M. Comunian, A. Pisent, and G. V. Lamanna, in Proceedings of the European Particle Accelerator Conference, Vienna, 2000 (EPS, Geneva, 2000), p. 927.

[38] S. N. Fu, S. X. Fang, X. L. Guan et al., in Proceedings of Linac 2006, Knoxville, Tennessee, USA (2006), pp. 165-167.

[39] K. R. Crandall et al., RFQ Design Codes, LANL Report No. LA-UR-96, 1998.

[40] http://laacg1.lanl.gov/laacg/services/download_sf.phtml.

[41] Chuan Zhang, Marco Busch, Florian Dziuba, Horst Klein, Holger Podlech, and Ulrich Ratzinger, Proceedings of IPAC2010, Kyoto, Japan (2010).

[42] P. A.P. Nghiem, N. Chauvin, M. Comunian, O. Delferriere, R. Duperrier, A. Mosnier, C. Oliver, and D. Uriot, Design report: Beam dynamics studies for the IFMIF-EVEDA accelerators, WBS: 4.3.2 and 4.2.4.

[43] I. Gonin et al., Proceedings of IPAC2010, Kyoto, Japan (2010), pp. 3022-3025.

[44] T. Tajima, in Proceedings of the 19th Particle Accelerator Conference, Chicago, Illinois, 2001 (Ref. [11]), pp. 903-905.
[45] T. Tajima et al., in Proceedings of the 20th Particle Accelerator Conference, Portland, OR, 2003 (Ref. [12]), pp. 1341-1343.

[46] Y.L. Chi et al., Proceedings of IPAC2013, Shanghai, China (2013).

[47] F. Gerigk, Proceedings of the 2002 Joint USPAS-CASJapan-Russia Accelerator School (2002), pp. 257-288.

[48] Li Zhi-hui, Tang Jing-Yu, Yan Fang, Geng Hui-Ping, Meng Cai, Sun Biao, Cheng Peng, Guo Zhen, and Sun Ji-LeiChinese Phys. C 37, 037005 (2013).

[49] M. Johnson et al., in Proceedings of the 21st Particle Accelerator Conference, Knoxville, 2005 (Ref. [29]).

[50] N. Solyak et al., in Proceedings of the 25th International Linear Accelerator Conference LINAC10, Tsukuba, Japan (KEK, Tsukuba, Japan, 2010), pp. 674-678.

[51] Z. Guo, Master thesis, University of Chinese Academy of Sciences, 2013.

[52] Z. Guo, H.P. Geng, Z.H. Li, and J.Y. Tang, in Proceedings of HB2012, Beijing, China, 2012.

[53] F. Gerigk and I. Hofmann, in Proceedings of the 19th Particle Accelerator Conference, Chicago, Illinois, 2001 (Ref. [11]).

[54] M. Reiser and N. Brown, Phys. Rev. Lett. 74, 1111 (1995).

[55] I. Hofmann and G. Franchetti, in Proceedings of the 8th European Particle Accelerator Conference, Paris, 2002 (EPS-IGA and CERN, Geneva, 2002), pp. 74-78.

[56] P. Pierini, in Proceedings of the HPPA5 Workshop, Mol, 2007.

[57] J. Galambos et al., in Proceedings of the Fifth International Workshop on the Utilisation and Reliability of High Power Proton Accelerators, Mol, Belgium, 2007.

[58] B. Sun et al., in Proceedings of HB2012, Beijing, China (2012).

[59] P. N. Ostroumov, V. N. Aseev, and B. Mustapha, Phys. Rev. ST Accel. Beams 7, 090101 (2004).

[60] F. Gerigk et al., CERN Report No. CERN.2006.006 (2006).

[61] X.-Y. Wu (private communication).

[62] H. Jungwirth, Conceptual Design Report-The Superconducting Injector Linac for the Cooler Synchrotron COSY, 2002, p. 22.

[63] B. Aune et al., Technical Report of ESS - The linac, Vol. III, (2002), pp. 111-114.

[64] S. Sato et al., in Proceedings of LINAC 2004, Lübeck, Germany (2004), pp. 429-431.

[65] B. Mustapha, V.N.Aseev, and P.N. Ostroumov, in Proceedings of the 23rd Particle Accelerator Conference, Vancouver, Canada, 2009 (Ref. [7]).

[66] M. Champion, in Proceedings of the 2007 Particle Accelerator Conference, Albuquerque, New Mexico (IEEE, New York, 2007). 